Research Article

\title{
Extensions of Dombi Aggregation Operators for Decision Making under $m$-Polar Fuzzy Information
}

\author{
Muhammad Akram $\left(\mathbb{D},{ }^{1}\right.$ Naveed Yaqoob, ${ }^{2}$ Ghous Ali, ${ }^{1}$ and Wathek Chammam ${ }^{3}{ }^{3}$ \\ ${ }^{1}$ Department of Mathematics, University of the Punjab, New Campus, Lahore, Pakistan \\ ${ }^{2}$ Department of Mathematics and Statistics, Riphah International University, I-14, Islamabad, Pakistan \\ ${ }^{3}$ Department of Mathematics, College of Science, Al Zulfi, Majmaah University, P.O. Box 66, Al-Majmaah 11952, Saudi Arabia
}

Correspondence should be addressed to Wathek Chammam; w.chammam@mu.edu.sa

Received 12 June 2020; Accepted 6 July 2020; Published 1 August 2020

Academic Editor: Tahir Mahmood

Copyright (c) 2020 Muhammad Akram et al. This is an open access article distributed under the Creative Commons Attribution License, which permits unrestricted use, distribution, and reproduction in any medium, provided the original work is properly cited.

\begin{abstract}
An $m$-polar fuzzy set is a powerful mathematical model to analyze multipolar, multiattribute, and multi-index data. The $m$-polar fuzzy sets have appeared as a useful tool to portray uncertainty in multiattribute decision making. The purpose of this article is to analyze the aggregation operators under the $m$-polar fuzzy environment with the help of Dombi norm operations. In this article, we develop some averaging and geometric aggregation operators using Dombi $t$-norm and $t$-conorm to handle uncertainty in $m$-polar fuzzy $(m \mathrm{~F}$, henceforth) information, which are $m \mathrm{~F}$ Dombi weighted averaging $(m \mathrm{FDWA})$ operator, $m \mathrm{~F}$ Dombi ordered weighted averaging $(m$ FDOWA) operator, $m$ F Dombi hybrid averaging $(m$ FDHA) operator, $m$ F Dombi weighted geometric $(m$ FDWG) operator, $m$ F Dombi weighted ordered geometric operator, and $m$ F Dombi hybrid geometric $(m$ FDHG) operator. We investigate properties, namely, idempotency, monotonicity, and boundedness, for the proposed operators. Moreover, we give an algorithm to solve multicriteria decision-making issues which involve $m$ F information with $m$ FDWA and $m$ FDWG operators. To prove the validity and feasibility of the proposed model, we solve two numerical examples with our proposed models and give comparison with $m$ F-ELECTRE-I approach (Akram et al. 2019) and $m$ F Hamacher aggregation operators (Waseem et al. 2019). Finally, we check the effectiveness of the developed operators by a validity test.
\end{abstract}

\section{Introduction}

Multicriteria decision making (MCDM) is performing a vital role in different areas, including social, physical, medical, and environmental sciences. MCDM methods are not only used to determine a suitable object but also used to rank the objects in an appointed problem. To solve different uncertain problems for decision making, Atanassov [1] presented the concept of intuitionistic fuzzy set (IFS) which considers both membership and nonmembership parts, an extension of fuzzy set [2] in which simple membership part is characterized.

Aggregation operators (AOs) perform an important role in order to combine data into a single form and solve MCDM problems. For example, Yager [3] introduced weighted AOs. $\mathrm{Xu}$ [4] proposed some new AOs under
IFSs. Xu and Yager [5] developed certain new geometric AOs and solved some real-world MCDM problems. From the inspection of an object, it can be easily seen that there exist two properties of the object which are opposite to each other. With this perspective, Zhang [6] presented the idea of bipolar fuzzy set (BFS). BFSs provide generalized structure as compared to fuzzy sets [2] whose memberships belong to $[-1,0] \times[0,1]$. Bipolarity plays an important role in different research areas and provides more flexibility as compared to the fuzzy methods. In the last decades, a lot of researchers, attracted by this efficient concept, applied it to aggregate bipolar information using different $t$-norms and their corresponding conorms, including Hamacher and Dombi $t$-norms and their corresponding conorms. For example, Wei et al. [7] developed some bipolar fuzzy Hamacher weighted 
averaging and geometric AOs. By combining Hamacher operations and prioritized AOs, Gao et al. [8] proposed dual hesitant bipolar fuzzy Hamacher prioritized weighted AOs and applied the proposed methodologies to an MCDM problem. Liu [9] utilized interval-valued intuitionistic fuzzy numbers with Hamacher AOs and developed multicriteria methods for group decision making. Jana et al. [10] applied weighted, ordered weighted, and hybrid average and geometric AOs for the aggregation of bipolar fuzzy information using Dombi $t$-conorm and $t$-norm. They also proposed bipolar fuzzy Dombi prioritized AOs in [11]. He [12] developed hesitant fuzzy Dombi AOs and investigated typhoon disaster assessment using proposed theory. Xu and Wei [13] introduced different dual hesitant bipolar fuzzy AOs to solve MCDM problems. Xu [14] proposed intuitionistic fuzzy power AOs for multiattribute group decision making. Xiao [15] constructed induced interval-valued intuitionistic fuzzy Hamacher AOs and discussed their application to MCDM. Chen and Ye [16] discussed MCDM problem under Dombi operations in singlevalued neutrosophic situation. Garg [17] presented some generalized interactive AOs under Einstein operations in Pythagorean fuzzy environment and discussed a decision-making issue. Akram et al. [18] proposed different Pythagorean Dombi fuzzy AOs and studied their applications in MCDM. Shahzadi et al. [19] introduced Pythagorean fuzzy Yager AOs for decision making. Peng and Yang [20] investigated different basic properties of interval-valued Pythagorean fuzzy AOs. Wang et al. [21] introduced some new types of q-rung orthopair fuzzy Hamy mean AOs to handle MCDM situations. Arora and Garg [22] proposed robust AOs with an intuitionistic fuzzy soft environment. Wang and Li [23] developed Pythagorean fuzzy interaction power Bonferroni mean AOs and discussed their applications to MCDM. Chiclana et al. [24] introduced some ordered weighted geometric operators and solved a decision-making problem. Liang et al. [25] developed Pythagorean fuzzy Bonferroni mean AOs.

Nowadays, experts believe that multipolarity performs a vital role in many practical situations. Due to the presence of multipolar data in different daily life problems of science and technology, Chen et al. [26] initiated the notion of $m \mathrm{~F}$ set theory as generalization of fuzzy and bipolar fuzzy sets. Waseem et al. [27] studied recently MCDM problems based on $m \mathrm{~F}$ Hamacher AOs. Khameneh and Kilicman [28] proposed $m \mathrm{~F}$ soft weighted AOs and applied these AOs in decision making. In view of the fact that $m \mathrm{~F}$ sets have an efficient strength to handle vague data which arise in several real-life problems, in this paper, we generalize Dombi AOs to aggregate the $m \mathrm{~F}$ information. The study of $\mathrm{AOs}$ under Dombi operations is very popular. Thus, an efficient research topic is how to aggregate $m \mathrm{~F}$ numbers with Dombi operations. To tackle this dilemma, in this article, we present some $m \mathrm{~F}$ Dombi AOs on the ground of classical geometric, arithmetic, and Dombi operations. For more information and terminologies on AOs, the readers are referred to [29-45].

An $m \mathrm{~F}$ model is more general than the fuzzy sets and BFSs due to the wider range of applicability over different complex problems. The $m \mathrm{~F}$ sets can handle much more details about an element and can explain uncertainties concurrently more precisely than the other existing methods, like fuzzy set and BFS. The motivation of developed AOs is summarized as below.

(1) A very difficult MCDM problem is the estimation of the supreme option in an $m \mathrm{~F}$ environment due to the involvement of several imprecise factors. Assessment of information in different MCDM techniques is simply depicted through fuzzy and bipolar fuzzy numbers which may not consider all the data in a real-world problem.

(2) As a general theory, $m$ F numbers describe efficient execution in the assessment process about uncertain, imprecise, and vague multipolar information. Thus, $m \mathrm{~F}$ theory provides an excellent approach for the assessment of objects under multinary data.

(3) In view of the fact that Dombi AOs are simple but provide a pioneering tool for solving MCDM problems when combined with other powerful mathematical tools, this article aims to develop Dombi AOs in an $m \mathrm{~F}$ environment to handle complex problems.

(4) An $m \mathrm{~F}$ model is different from the mathematical tools like fuzzy sets and BFSs because the fuzzy set and BFS can only handle one-dimensional data and two-dimensional data, respectively, which may prompt a loss in data. Nevertheless, in many daily life problems, we handle the situations having higher dimension to sort out all the attributes and their subcharacteristics.

(5) The Dombi AOs employed in the construction of $m \mathrm{~F}$ Dombi AOs are more suitable than all other aggregation approaches to tackle the MCDM situations as developed AOs have ability to consider all the information within the aggregation procedure.

(6) Dombi AOs make the optimal outcomes more accurate and definite when utilized in practical MCDM problems under $m \mathrm{~F}$ environment.

(7) The proposed $m \mathrm{~F}$ Dombi operators handle the drawbacks of existing AOs, including bipolar fuzzy Dombi AOs [10].

Therefore, some $m \mathrm{~F}$ Dombi AOs are developed to choose the best option in different decision-making situations. The developed operators have some advantages over other approaches which are given as follows: 
(1) Our proposed methods explain the problems more accurately which involve multiple attributes because they consider $m \mathrm{~F}$ numbers.

(2) The developed AOs are more precise and efficient with single attribute.

(3) To solve practical problems by using Dombi AOs with $m \mathrm{~F}$ numbers is very significant.

The rest of this article is structured as follows. Section 2 recalls some fundamental definitions and operations of the $m \mathrm{~F}$ numbers ( $m \mathrm{FNs})$. Section 3 presents $m$ FDWA, $m$ FDOWA, $m$ FDHA, $m$ FDWG, $m$ FDOWG, and $m$ FDHG operators. Section 4 develops a methodology of these AOs to model $m$ F MCDM problems. Section 5 discusses two applications: first for the selection of best agricultural land and second for the selection of best commercial bank. Section 6 provides comparative analysis of developed approaches with $m$ F ELECTRE-I model [46] and $m$ F Hamacher AOs [27]. Section 7 discusses the conclusions and future directions.

\section{Preliminaries}

Definition 1 (see [26]). An $m \mathrm{~F}$ set on a universal set $U$ is a mapping $\zeta: U \longrightarrow[0,1]^{m}$. The membership of every object is described by $\zeta(u)=\left(p_{1} \circ \zeta(u), p_{2} \circ \zeta(u), \ldots, p_{m} \circ \zeta(u)\right)$ where $p_{r} \circ \zeta:[0,1]^{m} \longrightarrow[0,1]$ is the $r$-th projection mapping.

Let $\hat{\zeta}=\left(p_{1} \circ \zeta, \ldots, p_{m} \circ \zeta\right)$ be an $m \mathrm{FN}$, where $p_{r} \circ \zeta \in[0,1], \forall r=1,2, \ldots, m$. We define the score and accuracy functions of $\widehat{\zeta}$, respectively, as follows.

Definition 2 (see [27]). For an $m \mathrm{FN} \widehat{\zeta}=\left(p_{1} \circ \zeta, \ldots, p_{m} \circ \zeta\right)$, we define a score function $S$ as follows:

$$
S(\widehat{\zeta})=\frac{1}{m}\left(\sum_{r=1}^{m}\left(p_{r} \circ \zeta\right)\right), \quad S(\widehat{\zeta}) \in[0,1]
$$

Definition 3 (see [27]). For an $m \mathrm{FN} \widehat{\zeta}=\left(p_{1} \circ \zeta, \ldots, p_{m} \circ \zeta\right)$, an accuracy function $H$ is defined as

$$
H(\widehat{\zeta})=\frac{1}{m}\left(\sum_{r=1}^{m}(-1)^{r+1}\left(p_{r} \circ \zeta-1\right)\right), \quad H(\widehat{\zeta}) \in[-1,1] .
$$

From Definitions 2 and 3, it can be readily seen that for any $m \mathrm{FN} \widehat{\zeta}, S(\widehat{\zeta}) \in[0,1]$ and $H(\widehat{\zeta}) \in[-1,1]$. Notice that $H(\widehat{\zeta})$ represents the accuracy degree of $\widehat{\zeta}$. Thus, a higher value of $H(\widehat{\zeta})$ represents a higher accuracy degree for $m \mathrm{FN}$ $\widehat{\zeta}$.

Using Definitions 2 and 3, we now give the following ordered relation criteria for any two $m \mathrm{FNs}$.

Definition 4 (see [27]). Let $\widehat{\zeta}_{1}=\left(p_{1} \circ \zeta_{1}, \ldots, p_{m} \circ \zeta_{1}\right)$ and $\widehat{\zeta}_{2}=\left(p_{1} \circ \zeta_{2}, \ldots, p_{m} \circ \zeta_{2}\right)$ be two $m$ FNs. Then,
(1) $\widehat{\zeta}_{1}<\widehat{\zeta}_{2}, \quad$ if $S\left(\widehat{\zeta}_{1}\right)<S\left(\widehat{\zeta}_{2}\right)$

(2) $\widehat{\zeta}_{1}>\widehat{\zeta}_{2}, \quad$ if $S\left(\widehat{\zeta}_{1}\right)>S\left(\widehat{\zeta}_{2}\right)$.

(3) $\widehat{\zeta}_{1}=\widehat{\zeta}_{2}, \quad$ if $S\left(\widehat{\zeta}_{1}\right)=S\left(\widehat{\zeta}_{2}\right)$ and $H\left(\widehat{\zeta}_{1}\right)=H\left(\widehat{\zeta}_{2}\right)$.

(4) $\widehat{\zeta}_{1}<\widehat{\zeta}_{2}$, if $S\left(\widehat{\zeta}_{1}\right)=S\left(\widehat{\zeta}_{2}\right)$, but $H\left(\widehat{\zeta}_{1}\right)<H\left(\widehat{\zeta}_{2}\right)$.

(5) $\widehat{\zeta}_{1}>\widehat{\zeta}_{2}$, if $S\left(\widehat{\zeta}_{1}\right)=S\left(\widehat{\zeta}_{2}\right)$, but $H\left(\widehat{\zeta}_{1}\right)>H\left(\widehat{\zeta}_{2}\right)$.

Some basic operations for $m \mathrm{FNs}$ are given by [27]

(1) $\widehat{\zeta}_{1} \boxplus \widehat{\zeta}_{2}=\left(p_{1} \circ \zeta_{1}+p_{1} \circ \zeta_{2}-p_{1} \circ \zeta_{1} \cdot p_{1} \circ \zeta_{2}, \ldots, p_{m}\right.$ $\left.\circ \zeta_{1}+p_{m} \circ \zeta_{2}-p_{m} \circ \zeta_{1} \cdot p_{m} \circ \zeta_{2}\right)$.

(2) $\widehat{\zeta}_{1} \otimes \widehat{\zeta}_{2}=\left(p_{1} \circ \zeta_{1} \cdot p_{1} \circ \zeta_{2}, \ldots, p_{m} \circ \zeta_{1} \cdot p_{m} \circ \zeta_{2}\right)$.

(3) $\left.\beta \widehat{\zeta}=\left(1-\left(1-p_{1} \circ \zeta\right)^{\beta}\right), \ldots, 1-\left(1-p_{m} \circ \zeta\right)^{\beta}\right)$, $\beta>0$.

(4) $(\widehat{\zeta})^{\beta}=\left(\left(p_{1} \circ \zeta\right)^{\beta}, \ldots,\left(p_{m} \circ \zeta\right)^{\beta}\right), \quad \beta>0$.

(5) $\widehat{\zeta}^{c}=\left(1-p_{1} \circ \zeta, \ldots, 1-p_{m} \circ \zeta\right)$.

(6) $\hat{\zeta}_{1} \subseteq \widehat{\zeta}_{2}$, if and only if $p_{1} \circ \zeta_{1} \leq p_{1} \circ \zeta_{2}, \ldots, p_{m} \circ \zeta_{1} \leq p_{m} \circ \zeta_{2}$.

(7) $\widehat{\zeta}_{1} \cup \widehat{\zeta}_{2}=\left(\max \left(p_{1} \circ \zeta_{1}, p_{1} \circ \zeta_{2}\right), \ldots, \max \left(p_{m} \circ \zeta_{1}\right.\right.$ $\left.\left.p_{m} \circ \zeta_{2}\right)\right)$.

(8) $\widehat{\zeta}_{1} \cap \widehat{\zeta}_{2}=\left(\min \left(p_{1} \circ \zeta_{1}, p_{1} \circ \zeta_{2}\right), \ldots, \min \right.$ $\left.\left(p_{m} \circ \zeta_{1}, p_{m} \circ \zeta_{2}\right)\right)$

Theorem 1 (see [27]). For two mFNs $\widehat{\zeta}_{1}=\left(p_{1} \circ \zeta_{1}, \ldots, p_{m} \circ \zeta_{1}(u)\right)$ and $\widehat{\zeta}_{2}=\left(p_{1} \circ \zeta_{2}, \ldots, p_{m} \circ \zeta_{2}\right)$ with $\beta, \beta_{1}, \beta_{2}>0$, we have
(1) $\widehat{\zeta}_{1} \boxplus \widehat{\zeta}_{2}=\widehat{\zeta}_{2} \boxplus \widehat{\zeta}_{1}$.
(2) $\widehat{\zeta}_{1} \otimes \widehat{\zeta}_{2}=\widehat{\zeta}_{2} \otimes \widehat{\zeta}_{1}$.
(3) $\beta\left(\widehat{\zeta}_{1} \boxplus \widehat{\zeta}_{2}\right)=\beta\left(\widehat{\zeta}_{1}\right) \boxplus \beta\left(\widehat{\zeta}_{2}\right)$.
(4) $\left(\widehat{\zeta}_{1} \otimes \widehat{\zeta}_{2}\right)^{\beta}=\left(\widehat{\zeta}_{1}\right)^{\beta} \boxplus\left(\widehat{\zeta}_{2}\right)^{\beta}$.
(5) $\beta_{1} \widehat{\zeta}_{1} \boxplus \beta_{2} \widehat{\zeta}_{1}=\left(\beta_{1}+\beta_{2}\right) \widehat{\zeta}_{1}$.
(6) $\left(\widehat{\zeta}_{1}\right)^{\beta_{1}} \otimes\left(\widehat{\zeta}_{2}\right)^{\beta_{2}}=\left(\widehat{\zeta}_{1}\right)^{\beta_{1}+\beta_{2}}$
(7) $\left(\left(\widehat{\zeta}_{1}\right)^{\beta_{1}}\right)^{\beta_{2}}=\left(\widehat{\zeta}_{1}\right)^{\beta_{1} \beta_{2}}$.

Dombi [47] proposed operations, namely, Dombi sum $\oplus$ and Dombi product $\otimes$, which are, respectively, $t$-conorm and $t$-norm given by

$$
\begin{aligned}
& D^{*}(a, b)=a \oplus b=1-\frac{1}{1+\left\{(a / 1-a)^{k}+(b / 1-b)^{k}\right\}^{1 / k}}, \\
& D(a, b)=a \otimes b=\frac{1}{1+\left\{(1-a / a)^{k}+(1-b / b)^{k}\right\}^{1 / k}},
\end{aligned}
$$

where $k \geq 1$ and $a, b \in[0,1]$.

\section{3. $m$ F Dombi AOs}

In this section, we first give Dombi operations for $m$ FNs via Dombi $t$-conorm and Dombi $t$-norm and then we present $m \mathrm{~F}$ Dombi arithmetic and geometric AOs. Let $\widehat{\zeta}_{1}=\left(p_{1} \circ \zeta_{1}, \ldots, p_{m} \circ \zeta_{1}\right), \widehat{\zeta}_{2}=\left(p_{1} \circ \zeta_{2}, \ldots, p_{m} \circ \zeta_{2}\right)$ and $\zeta=\left(p_{1} \circ \zeta, \ldots, p_{m} \circ \zeta\right)$ be $m$ FNs. We give some fundamental Dombi operations of $m$ FNs as follows: 


$$
\begin{aligned}
\widehat{\zeta}_{1} \oplus \widehat{\zeta}_{2} & =\left(1-\frac{1}{1+\left\{\left(p_{1} \circ \zeta_{1} / 1-p_{1} \circ \zeta_{1}\right)^{k}+\left(p_{1} \circ \zeta_{2} / 1-p_{1} \circ \zeta_{2}\right)^{k}\right\}^{1 / k}}, \ldots, 1-\frac{1}{1+\left\{\left(p_{m} \circ \zeta_{1} / 1-p_{m} \circ \zeta_{1}\right)^{k}+\left(p_{m} \circ \zeta_{2} / 1-p_{m} \circ \zeta_{2}\right)^{k}\right\}^{1 / k}}\right) \\
\widehat{\zeta}_{1} \otimes \widehat{\zeta}_{2} & =\left(\frac{1}{1+\left\{\left(1-p_{1} \circ \zeta_{1} / p_{1} \circ \zeta_{1}\right)^{k}+\left(1-p_{1} \circ \zeta_{2} / p_{1} \circ \zeta_{2}\right)^{k}\right\}^{1 / k}}, \ldots, \frac{1}{1+\left\{\left(1-p_{m} \circ \zeta_{1} / p_{m} \circ \zeta_{1}\right)^{k}+\left(1-p_{m} \circ \zeta_{2} / p_{m} \circ \zeta_{2}\right)^{k}\right\}^{1 / k}}\right), \\
\hat{\beta} \widehat{\zeta} & =\left(1-\frac{1}{1+\left\{\beta\left(p_{1} \circ \zeta / 1-p_{1} \circ \zeta\right)^{k}\right\}^{1 / k}}, \ldots, 1-\frac{1}{1+\left\{\beta\left(p_{m} \circ \zeta / 1-p_{m} \circ \zeta\right)^{k}\right\}^{1 / k}}\right), \\
(\widehat{\zeta})^{\beta} & =\left(1-\frac{1}{1+\left\{\beta\left(1-p_{1} \circ \zeta / p_{1} \circ \zeta\right)^{k}\right\}^{1 / k}}, \ldots, 1-\frac{1}{1+\left\{\beta\left(1-p_{m} \circ \zeta / p_{m} \circ \zeta\right)^{k}\right\}^{1 / k}}\right),
\end{aligned}
$$

where $k>0$.

3.1. $m F$ Dombi Arithmetic AOs. We present $m \mathrm{~F}$ Dombi arithmetic AOs as follows.

Definition 5. For a collection of $m \mathrm{FNs}$ $\widehat{\zeta}_{j}=\left(p_{1} \circ \zeta_{j}, \ldots, p_{m} \circ \zeta_{j}\right)$ where $j=1,2, \ldots, n$, a mapping from $\zeta$ to $\zeta$ is called an $m$ FDWA operator, which is given by

$$
m \operatorname{FDWA}_{\Theta}\left(\widehat{\zeta}_{1}, \widehat{\zeta}_{2}, \ldots, \hat{\zeta}_{n}\right)=\oplus_{j=1}^{n}\left(\Theta_{j} \widehat{\zeta}_{j}\right),
$$

where $\Theta=\left(\Theta_{1}, \Theta_{2}, \ldots, \Theta_{n}\right)^{T}$ denotes the weights of $\widehat{\zeta}_{j}, \forall j=$ $1, \ldots, n$ and $\Theta_{j}>0$ with $\sum_{j=1}^{n} \Theta_{j}=1$.

We give the following theorem, which is used to apply the Dombi operations on $m$ FNs.

Theorem 2. For a collection of $m F N s$ $\widehat{\zeta}_{j}=\left(p_{1} \circ \zeta_{j}, \ldots, p_{m} \circ \zeta_{j}\right)$ where $j=1,2, \ldots, n$, an accumulated value of these mFNs using the mFDWA operators is defined as

$$
\begin{aligned}
\operatorname{mFDWA}_{\Theta}\left(\widehat{\zeta}_{1}, \hat{\zeta}_{2}, \ldots, \widehat{\zeta}_{n}\right) & =\oplus_{j=1}^{n}\left(\Theta_{j} \widehat{\zeta}_{j}\right), \\
& =\left(1-\frac{1}{\left.1+\left\{\sum_{j=1}^{n} \Theta_{j}\left(p_{1} \circ \zeta_{j} / 1-p_{1} \circ \zeta_{j}\right)^{k}\right\}^{1 / k}, \ldots, 1-\frac{1}{1+\left\{\sum_{j=1}^{n} \Theta_{j}\left(p_{m} \circ \zeta_{j} / 1-p_{m} \circ \zeta_{j}\right)^{k}\right\}^{1 / k}}\right)} .\right.
\end{aligned}
$$

Proof. We utilize the induction approach to show it.

Case 1. For $n=1$, by equation (6), we obtain

$$
\begin{aligned}
m \operatorname{FDWA}_{\Theta}\left(\widehat{\zeta}_{1}, \widehat{\zeta}_{2}, \ldots, \widehat{\zeta}_{n}\right) & =\Theta_{1} \widehat{\zeta}_{1}=\widehat{\zeta}_{1}, \quad\left(\text { since } \Theta_{1}=1\right) \\
& =\left(1-\frac{1}{1+\left\{\left(p_{1} \circ \zeta_{1} / 1-p_{1} \circ \zeta_{1}\right)^{k}\right\}^{1 / k}}, \ldots, 1-\frac{1}{1+\left\{\left(p_{m} \circ \zeta_{1} / 1-p_{m} \circ \zeta_{1}\right)^{k}\right\}^{1 / k}}\right)
\end{aligned}
$$

Hence, equation (6) satisfies when $n=1$.

Case 2. Now, we presume that equation (6) satisfies for $n=t$; here $t$ is an arbitrary natural number; then,

$$
\begin{aligned}
\operatorname{mFDWA}_{\Theta}\left(\widehat{\zeta}_{1}, \widehat{\zeta}_{2}, \ldots, \widehat{\zeta}_{t}\right) & =\oplus_{j=1}^{t}\left(\Theta_{j} \widehat{\zeta}_{j}\right) \\
& =\left(1-\frac{1}{1+\left\{\sum_{j=1}^{t} \Theta_{j}\left(p_{1} \circ \zeta_{j} / 1-p_{1} \circ \zeta_{j}\right)^{k}\right\}^{1 / k}}, \ldots, 1-\frac{1}{1+\left\{\sum_{j=1}^{t} \Theta_{j}\left(p_{m} \circ \zeta_{j} / 1-p_{m} \circ \zeta_{j}\right)^{k}\right\}^{1 / k}}\right)
\end{aligned}
$$

For $n=t+1$, 
$m \operatorname{FDWA}_{\Theta}\left(\widehat{\zeta}_{1}, \widehat{\zeta}_{2}, \ldots, \widehat{\zeta}_{t}, \widehat{\zeta}_{t+1}\right)=\oplus_{j=1}^{t}\left(\Theta_{j} \widehat{\zeta}_{j}\right) \oplus\left(\Theta_{t+1} \widehat{\zeta}_{t+1}\right)$,

$$
\begin{aligned}
& =\left(1-\frac{1}{1+\left\{\sum_{j=1}^{t} \Theta_{j}\left(p_{1} \circ \zeta_{j} / 1-p_{1} \circ \zeta_{j}\right)^{k}\right\}^{1 / k}}, \ldots, 1-\frac{1}{1+\left\{\sum_{j=1}^{t} \Theta_{j}\left(p_{m} \circ \zeta_{j} / 1-p_{m} \circ \zeta_{j}\right)^{k}\right\}^{1 / k}}\right) \\
& =\left(1-\frac{1}{1+\left\{\Theta_{t+1}\left(p_{1} \circ \zeta_{t+1} / 1-p_{1} \circ \zeta_{t+1}\right)^{k}\right\}^{1 / k}}, \ldots, 1-\frac{1}{1+\left\{\Theta_{t+1}\left(p_{m} \circ \zeta_{t+1} / 1-p_{m} \circ \zeta_{t+1}\right)^{k}\right\}^{1 / k}}\right) \\
& =\left(1-\frac{1}{1+\left\{\sum_{j=1}^{t+1} \Theta_{j}\left(p_{1} \circ \zeta_{j} / 1-p_{1} \circ \zeta_{j}\right)^{k}\right\}^{1 / k}}, \ldots, 1-\frac{1}{1+\left\{\sum_{j=1}^{t+1} \Theta_{j}\left(p_{m} \circ \zeta_{j} / 1-p_{m} \circ \zeta_{j}\right)^{k}\right\}^{1 / k}}\right) .
\end{aligned}
$$

Therefore, equation (6) satisfies for $n=t+1$. Hence, we deduce that equation (6) satisfies for every natural number $n$.

$$
\begin{aligned}
\operatorname{mFDWA}_{\Theta}\left(\widehat{\zeta}_{1}, \widehat{\zeta}_{2}, \widehat{\zeta}_{3}\right)= & \Theta_{j=1}^{3}\left(\Theta_{j} \widehat{\zeta}_{j}\right) \\
= & \left(1-\frac{1}{1+\left\{\sum_{j=1}^{n} \Theta_{j}\left(p_{1} \circ \zeta_{j} / 1-p_{1} \circ \zeta_{j}\right)^{k}\right\}^{1 / k}}, \ldots, 1-\frac{1}{1+\left\{\sum_{j=1}^{n} \Theta_{j}\left(p_{m} \circ \zeta_{j} / 1-p_{m} \circ \zeta_{j}\right)^{k}\right\}}\right) \\
= & \left(1-\frac{1}{1+\left(0.2 \times(0.4 / 1-0.4)^{3}+0.3 \times(0.3 / 1-0.3)^{3}+0.1 \times(0.7 / 1-0.7)^{3}+0.4 \times(0.5 / 1-0.5)^{3}\right)^{1 / 3}},\right. \\
& 1-\frac{1}{1+\left(0.2 \times(0.3 / 1-0.3)^{3}+0.3 \times(0.5 / 1-0.5)^{3}+0.1 \times(0.2 / 1-0.2)^{3}+0.4 \times(0.4 / 1-0.4)^{3}\right)^{1 / 3}}, \\
& \left.1-\frac{1}{1+\left(0.2 \times(0.8 / 1-0.8)^{3}+0.3 \times(0.1 / 1-0.1)^{3}+0.1 \times(0.4 / 1-0.4)^{3}+0.4 \times(0.6 / 1-0.6)^{3}\right)^{1 / 3}}\right), \\
= & (0.5467,0.4312,0.7076) .
\end{aligned}
$$

We now explore some useful laws of $m$ FDWA operators as follows.

Theorem 3 (idempotent law). Let $\widehat{\zeta}_{j}=\left(p_{1} \circ \zeta_{j}, \ldots, p_{m} \circ \zeta_{j}\right)$ be a family of " $n$ " mFNs, which are equal, i.e., $\widehat{\zeta}_{j}=\zeta$; then,
Example 1. Let $\widehat{\zeta}_{1}=(0.4,0.3,0.8), \widehat{\zeta}_{2}=(0.3,0.5,0.1), \widehat{\zeta}_{3}=$ $(0.7,0.2,0.4)$, and $\hat{\zeta}_{4}=(0.5,0.4,0.6)$ be $3 \mathrm{FNs}$ and $\Theta=(0.2,0.3,0.1,0.4)^{T}$ be weights related to these $3 \mathrm{FNs}$. Then, for $k=3$, 


$$
\begin{aligned}
m \operatorname{FDWA}_{\Theta}\left(\widehat{\zeta}_{1}, \widehat{\zeta}_{2}, \ldots, \widehat{\zeta}_{n}\right) & =\oplus_{j=1}^{n}\left(\Theta_{j} \widehat{\zeta}_{j}\right) \\
& =\left(1-\frac{1}{1+\left\{\sum_{j=1}^{n} \Theta_{j}\left(p_{1} \circ \zeta_{j} / 1-p_{1} \circ \zeta_{j}\right)^{k}\right\}^{1 / k}}, \ldots, 1-\frac{1}{1+\left\{\sum_{j=1}^{n} \Theta_{j}\left(p_{m} \circ \zeta_{j} / 1-p_{m} \circ \zeta_{j}\right)^{k}\right\}^{1 / k}}\right) \\
& =\left(1-\frac{1}{1+\left\{\left(p_{1} \circ \zeta / 1-p_{1} \circ \zeta\right)^{k}\right\}^{1 / k}}, \ldots, 1-\frac{1}{1+\left\{\left(p_{m} \circ \zeta / 1-p_{m} \circ \zeta\right)^{k}\right\}^{1 / k}}\right) \\
& =\left(p_{1} \circ \zeta, p_{2} \circ \zeta, \ldots, p_{m} \circ \zeta\right), \quad \text { for } k=1 \\
& =\widehat{\zeta} .
\end{aligned}
$$

Hence, $\operatorname{mFDWA}_{\Theta}\left(\widehat{\zeta}_{1}, \widehat{\zeta}_{2}, \ldots, \widehat{\zeta}_{n}\right)=\widehat{\zeta}$ holds if $\widehat{\zeta}_{j}=\widehat{\zeta}$, for all " $j$ " varies from 1 to $n$.

Theorem 4 (bounded law). Let $\widehat{\zeta}_{j}=\left(p_{1} \circ \zeta_{j}, \ldots, p_{m} \circ \zeta_{j}\right)$ be a collection of " $n$ " mFNs, $\widehat{\zeta}^{-}=\cap_{j=1}^{n}\left(\zeta_{j}\right)$, and $\hat{\zeta}^{+}=\cup_{j=1}^{n}\left(\zeta_{j}\right)$; then,

$$
\widehat{\zeta}^{-} \leq m \operatorname{FDWA}_{\Theta}\left(\widehat{\zeta}_{1}, \widehat{\zeta}_{2}, \ldots, \widehat{\zeta}_{n}\right) \leq \hat{\zeta}^{+}
$$

Theorem 5 (monotonic law). For two collections of $m F N s \widehat{\zeta}_{j}$ and $\widehat{\zeta}_{j}^{\prime}, j=1,2, \ldots, n$, if $\widehat{\zeta}_{j} \leq \widehat{\zeta}_{j}^{\prime}$,

$$
m \operatorname{FDWA}_{\Theta}\left(\widehat{\zeta}_{1}, \widehat{\zeta}_{2}, \ldots, \widehat{\zeta}_{n}\right) \leq m \operatorname{FDWA}_{\Theta}\left(\widehat{\zeta}_{1}^{\prime}, \widehat{\zeta}_{2}^{\prime}, \ldots, \widehat{\zeta}_{n}^{\prime}\right) .
$$

Definition 6. For a collection of $m \mathrm{FNs}$ $\widehat{\zeta}_{j}=\left(p_{1} \circ \zeta_{j}, \ldots, p_{m} \circ \zeta_{j}\right), j=1,2, \ldots, n$, an $m$ FDOWA operator is a function $m$ FDOWA: $\widehat{\zeta}^{n} \longrightarrow \widehat{\zeta}$, which is given by

$$
\operatorname{mFDOWA}_{w}\left(\widehat{\zeta}_{1}, \widehat{\zeta}_{2}, \ldots, \widehat{\zeta}_{n}\right)=\oplus_{j=1}^{n}\left(w_{j} \widehat{\zeta}_{\sigma(j)}\right)
$$

where $w=\left(w_{1}, w_{2}, \ldots, w_{n}\right)^{T}$ denotes the weights and $w_{j} \in(0,1]$ with $\sum_{j=1}^{n} w_{j}=1 . \sigma(j),(j=1,2, \ldots, n)$ represents the permutation, for which $\widehat{\zeta}_{\sigma(j-1)} \geq \widehat{\zeta}_{\sigma(j)}$.

Theorem 6. For a collection of $m F N s$ $\widehat{\zeta}_{j}=\left(p_{1} \circ \zeta_{j}, \ldots, p_{m} \circ \zeta_{j}\right)$ where $j=1,2, \ldots, n$, an accumulated value of these mFNs using the mFDOWA operators is defined as

Now, we present mFDOWA operator.

$$
\begin{aligned}
\operatorname{mFDOWA}_{w}\left(\widehat{\zeta}_{1}, \widehat{\zeta}_{2}, \ldots, \widehat{\zeta}_{n}\right) & =\oplus_{j=1}^{n}\left(w_{j} \widehat{\zeta}_{\sigma(j)}\right) \\
& =\left(1-\frac{1}{1+\left\{\sum_{j=1}^{n} w_{j}\left(p_{1} \circ \zeta_{\sigma(j)} / 1-p_{1} \circ \zeta_{\sigma(j)}\right)^{k}\right\}^{1 / k}}, \ldots, 1-\frac{1}{1+\left\{\sum_{j=1}^{n} w_{j}\left(p_{m} \circ \zeta_{\sigma(j)} / 1-p_{m} \circ \zeta_{\sigma(j)}\right)^{k}\right\}^{1 / k}}\right)
\end{aligned}
$$

Example 2. Let $\widehat{\zeta}_{1}=(0.4,0.5,0.3,0.8), \widehat{\zeta}_{2}=(0.3,0.4,0.1$, $0.7)$, and $\widehat{\zeta}_{3}=(0.8,0.7,0.6,0.4)$ be $4 \mathrm{FNs}$ with weights $w=$ $(0.3,0.1,0.6)^{T}$. Then, for $k=3$, we compute the score values as

$$
\begin{aligned}
& S\left(\widehat{\zeta}_{1}\right)=\frac{0.4+0.5+0.3+0.8}{4}=0.5, \\
& S\left(\widehat{\zeta}_{2}\right)=\frac{0.3+0.4+0.1+0.7}{4}=0.375 \\
& S\left(\widehat{\zeta}_{3}\right)=\frac{0.8+0.7+0.6+0.4}{4}=0.625 .
\end{aligned}
$$

$$
\begin{aligned}
\text { Since } S\left(\widehat{\zeta}_{3}\right)>S\left(\widehat{\zeta}_{1}\right)>S\left(\widehat{\zeta}_{2}\right) \\
\widehat{\zeta}_{\sigma(1)}=\widehat{\zeta}_{3}=(0.8,0.7,0.6,0.4) \\
\widehat{\zeta}_{\sigma(2)}=\widehat{\zeta}_{1}=(0.4,0.5,0.3,0.8) \\
\widehat{\zeta}_{\sigma(3)}=\widehat{\zeta}_{2}=(0.3,0.4,0.1,0.7)
\end{aligned}
$$

Then, from Definition 6, 


$$
\begin{aligned}
& \operatorname{mFDOWA}_{w}\left(\widehat{\zeta}_{1}, \widehat{\zeta}_{2}, \widehat{\zeta}_{3}\right)=\oplus_{j=1}^{3}\left(w_{j} \widehat{\zeta}_{\sigma(j)}\right) \\
& =\left(1-\frac{1}{1+\left\{\sum_{j=1}^{n} w_{j}\left(p_{1} \circ \zeta_{\sigma(j)} / 1-p_{1} \circ \zeta_{\sigma(j)}\right)^{k}\right\}^{1 / k}}, \ldots, 1-\frac{1}{1+\left\{\sum_{j=1}^{n} w_{j}\left(p_{m} \circ \zeta_{\sigma(j)} / 1-p_{m} \circ \zeta_{\sigma(j)}\right)^{k}\right\}^{1 / k}}\right), \\
& =\left(1-\frac{1}{1+\left(0.3 \times(0.8 / 1-0.8)^{3}+0.1 \times(0.4 / 1-0.4)^{3}+0.6 \times(0.3 / 1-0.3)^{3}\right)^{1 / 3}},\right. \\
& 1-\frac{1}{1+\left(0.3 \times(0.7 / 1-0.7)^{3}+0.1 \times(0.5 / 1-0.5)^{3}+0.6 \times(0.4 / 1-0.4)^{3}\right)^{1 / 3}}, \\
& 1-\frac{1}{1+\left(0.3 \times(0.6 / 1-0.6)^{3}+0.1 \times(0.3 / 1-0.3)^{3}+0.6 \times(0.1 / 1-0.1)^{3}\right)^{1 / 3}}, \\
& \left.1-\frac{1}{1+\left(0.3 \times(0.4 / 1-0.4)^{3}+0.1 \times(0.8 / 1-0.8)^{3}+0.6 \times(0.7 / 1-0.7)^{3}\right)^{1 / 3}}\right), \\
& =(0.7284,0.6152,0.5017,0.7073) \text {. }
\end{aligned}
$$

Remark 1 . Note that $m$ FDOWA operators satisfy properties, namely, idempotency, boundedness, and monotonicity, as described in Theorems 3, 4, and 5.

Theorem 7 (commutative law). For any two collections of $m F N s \widehat{\zeta}_{j}$ and $\widehat{\zeta}_{j}^{\prime} j=1,2, \ldots, n$, we get

$$
m \operatorname{FDOWA}_{w}\left(\widehat{\zeta}_{1}, \widehat{\zeta}_{2}, \ldots, \widehat{\zeta}_{n}\right)=m \operatorname{FDOW} \zeta_{w}\left(\hat{\zeta}_{1}^{\prime}, \hat{\zeta}_{2}^{\prime}, \ldots, \hat{\zeta}_{n}^{\prime}\right) \text {, }
$$

where $\hat{\zeta}_{j}^{\prime}$ is any permutation of $\widehat{\zeta}_{j}$.

We see that $m F D W A$ and $m F D O W A$ operators aggregate weighted $\mathrm{mFNs}$ and their ordering, respectively. Now, we develop a novel operator called mFDHA operator, which obtains the properties of both mFDWA and mFDOWA operators.

Definition 7. For a family of $m \mathrm{FNs}$ $\widehat{\zeta}_{j}=\left(p_{1} \circ \zeta_{j}, p_{2} \circ \zeta_{j}, \ldots, p_{m} \circ \zeta_{j}\right), j=1,2, \ldots, n$, an $m$ FDHA operator is defined as

$$
m \operatorname{FDHA}_{w, \Theta}\left(\widehat{\zeta}_{1}, \widehat{\zeta}_{2}, \ldots, \widehat{\zeta}_{n}\right)=\oplus_{j=1}^{n}\left(w_{j} \widetilde{\widetilde{\zeta}}_{\sigma(j)}\right)
$$

where $w=\left(w_{1}, w_{2}, \ldots, w_{n}\right)^{T}$ denotes the weights corresponding to the $m$ FNs $\widehat{\zeta}_{j}$ with the conditions $w_{j} \in(0,1], \sum_{j=1}^{n} w_{j}=1, \widehat{\zeta}_{\sigma(j)}$ is the $j$ th biggest $m \mathrm{FN}$, $\widetilde{\widetilde{\zeta}}_{\sigma(j)}=\left(n \Theta_{j}\right) \widehat{\zeta}_{j},(j=1,2, \ldots, n)$, and $\Theta=\left(\Theta_{1}, \Theta_{2}, \ldots, \Theta_{n}\right)$ is a vector having weights, with $\Theta_{j} \in(0,1], \sum_{j=1}^{n} \Theta_{j}=1$.

Notice that when $w=((1 / n),(1 / n), \ldots,(1 / n)), m$ FDHA operator converts into mFDWA operator. If $\Theta=((1 / n),(1 / n), \ldots,(1 / n))$, then mFDHA operator becomes $m$ FDOWA operator. Thus, $m$ FDHA operator is a generalization for both operators, $m$ FDWA and $m$ FDOWA, which describes the degrees and ordering of $m \mathrm{FNs}$.

The following theorem can be readily showed by same steps as in Theorem 2 .

Theorem 8. For a collection of $m F N s$ $\widehat{\zeta}_{j}=\left(p_{1} \circ \zeta_{j}, \ldots, p_{m} \circ \zeta_{j}\right)$ where $j=1,2, \ldots, n$, an accumulated score of these mFNs using the mFDHA operators is defined as

$$
\begin{aligned}
\operatorname{mFDHA}_{w, \Theta}\left(\widehat{\zeta}_{1}, \widehat{\zeta}_{2}, \ldots, \widehat{\zeta}_{n}\right) & =\oplus_{j=1}^{n}\left(w_{j} \widetilde{\widehat{\zeta}}_{\sigma(j)}\right), \\
& =\left(1-\frac{1}{\left.1+\left\{\sum_{j=1}^{n} w_{j}\left(p_{1} \circ \zeta_{\sigma(j)} / 1-p_{1} \circ \zeta_{\sigma(j)}\right)^{k}\right\}^{1 / k}, \ldots, 1-\frac{1}{1+\left\{\sum_{j=1}^{n} w_{j}\left(p_{m} \circ \zeta_{\sigma(j)} / 1-p_{m} \circ \zeta_{\sigma(j)}\right)^{k}\right\}^{1 / k}}\right)} .\right.
\end{aligned}
$$


Example 3. Let $\widehat{\zeta}_{1}=(0.7,0.3,0.5), \widehat{\zeta}_{2}=(0.2,0.5,0.7)$, $\widehat{\zeta}_{3}=(0.8,0.2,0.1)$, and $\widehat{\zeta}_{4}=(0.6,0.7,0.9)$ be $3 \mathrm{FNs}$ with $w=(0.2,0.3,0.1,0.4)^{T}$, a weight vector corresponding to given 3FNs, and a vector $\Theta=(0.3,0.1,0.4,0.2)^{T}$ having weights. Then, by Definition 7 , for $k=3$,

$$
\begin{aligned}
\widetilde{\widetilde{\zeta}}_{1} & =\left(1-\frac{1}{1+\left\{n \Theta_{1}\left(p_{1} \circ \zeta_{1} / 1-p_{1} \circ \zeta_{1}\right)^{k}\right\}^{1 / k}}, \ldots, 1-\frac{1}{1+\left\{n \Theta_{1}\left(p_{3} \circ \zeta_{1} / 1-p_{3} \circ \zeta_{1}\right)^{k}\right\}^{1 / k}}\right), \\
& =\left(1-\frac{1}{1+\left(4 \times 0.3 \times(0.7 / 1-0.7)^{3}\right)^{1 / 3}}, 1-\frac{1}{1+\left(4 \times 0.3 \times(0.3 / 1-0.3)^{3}\right)^{1 / 3}}, 1-\frac{1}{1+\left(4 \times 0.3 \times(0.5 / 1-0.5)^{3}\right)^{1 / 3}}\right), \\
& =(0.7126,0.3129,0.5152) .
\end{aligned}
$$

Similarly,

$$
\begin{aligned}
\widetilde{\widetilde{\zeta}}_{2} & =\left(1-\frac{1}{1+\left(4 \times 0.1 \times(0.2 / 1-0.2)^{3}\right)^{1 / 3}}, 1-\frac{1}{1+\left(4 \times 0.1 \times(0.5 / 1-0.5)^{3}\right)^{1 / 3}}, 1-\frac{1}{1+\left(4 \times 0.1 \times(0.7 / 1-0.7)^{3}\right)^{1 / 3}}\right), \\
& =(0.1555,0.4242,0.6322), \\
\widetilde{\widetilde{\zeta}}_{3} & =\left(1-\frac{1}{1+\left(4 \times 0.4 \times(0.8 / 1-0.8)^{3}\right)^{1 / 3}}, 1-\frac{1}{1+\left(4 \times 0.4 \times(0.2 / 1-0.2)^{3}\right)^{1 / 3}}, 1-\frac{1}{1+\left(4 \times 0.4 \times(01 / 1-0.1)^{3}\right)^{1 / 3}}\right), \\
& =(0.8239,0.2262,0.1150),
\end{aligned}
$$$$
\widetilde{\zeta}_{4}=\left(1-\frac{1}{1+\left(4 \times 0.2 \times(0.6 / 1-0.6)^{3}\right)^{1 / 3}}, 1-\frac{1}{1+\left(4 \times 0.2 \times(0.7 / 1-0.7)^{3}\right)^{1 / 3}}, 1-\frac{1}{1+\left(4 \times 0.2 \times(0.9 / 1-0.9)^{3}\right)^{1 / 3}}\right)
$$$$
=(0.5820,0.6842,0.8931)
$$

Then, scores of $m$ FNs for $k=3$ are calculated as

$$
\begin{aligned}
& S\left(\widetilde{\zeta}_{1}\right)=\frac{0.7126+0.3129+0.5152}{3}=0.5136, \\
& S\left(\widetilde{\zeta}_{2}\right)=\frac{0.1555+0.4242+0.6322}{3}=0.4040, \\
& S\left(\widetilde{\zeta}_{3}\right)=\frac{0.8239+0.2262+0.1150}{3}=0.3884, \\
& S\left(\widetilde{\zeta}_{4}\right)=\frac{0.5820+0.6842+0.8931}{3}=0.7198 .
\end{aligned}
$$

Since $S\left(\widetilde{\widetilde{\zeta}}_{4}\right)>S\left(\widetilde{\widetilde{\zeta}}_{1}\right)>S\left(\widetilde{\widetilde{\zeta}}_{2}\right)>S\left(\widetilde{\widetilde{\zeta}}_{3}\right)$,

$$
\begin{aligned}
& \widetilde{\widetilde{\zeta}}_{\sigma(1)}=\widehat{\zeta}_{4}=(0.5820,0.6842,0.8931) \\
& \widetilde{\widetilde{\zeta}}_{\sigma(2)}=\widehat{\zeta}_{1}=(0.7126,0.3129,0.5152) \\
& \widetilde{\widetilde{\zeta}}_{\sigma(3)}=\widehat{\zeta}_{2}=(0.1555,0.4242,0.6322) \\
& \widetilde{\zeta}_{\sigma(4)}=\widehat{\zeta}_{3}=(0.8239,0.2262,0.1150)
\end{aligned}
$$

Then, from Theorem 8, 


$$
\begin{aligned}
m \mathrm{FDHA}_{w, \Theta}\left(\widehat{\zeta}_{1}, \widehat{\zeta}_{2}, \widehat{\zeta}_{3}, \widehat{\zeta}_{4}\right)= & \oplus_{j=1}^{4}\left(w_{j} \widehat{\zeta}_{\sigma(j)}\right) \\
= & \left(1-\frac{1}{1+\left\{\sum_{j=1}^{4} w_{j}\left(p_{1} \circ \zeta_{\sigma(j)} / 1-p_{1} \circ \zeta_{\sigma(j)}\right)^{k}\right\}^{1 / k}}, \ldots, 1-\frac{1}{1+\left\{\sum_{j=1}^{4} w_{j}\left(p_{3} \circ \zeta_{\sigma(j)} / 1-p_{3} \circ \zeta_{\sigma(j)}\right)^{k}\right\}^{1 / k}}\right), \\
= & \left(1-\frac{1}{1+\left(0.2 \times(0.5820 / 1-0.5820)^{3}+0.3 \times(0.7126 / 1-0.7126)^{3}+0.1 \times(0.1555 / 1-0.1555)^{3}+0.4 \times(0.8239 / 1-0.8239)^{3}\right)^{1 / 3}},\right. \\
& 1-\frac{1}{1+\left(0.2 \times(0.6842 / 1-0.6842)^{3}+0.3 \times(0.3129 / 1-0.3129)^{3}+0.1 \times(0.4242 / 1-0.4242)^{3}+0.4 \times(0.2262 / 1-0.2262)^{3}\right)^{1 / 3},} \\
& \left.1-\frac{1}{1+\left(0.2 \times(0.8931 / 1-0.8931)^{3}+0.3 \times(0.5152 / 1-0.5152)^{3}+0.1 \times(0.6322 / 1-0.6322)^{3}+0.4 \times(0.1150 / 1-0.1150)^{3}\right)^{1 / 3}}\right), \\
= & (0.7819,0.5620,0.8304) .
\end{aligned}
$$

3.2. $m F$ Dombi Geometric AOs. We now propose different types of Dombi geometric AOs with $m$ FNs, namely, $m$ FDWG operator, $m$ FDOWG operator, and $m$ FDHG operator.

Definition 8. For a family of $m \mathrm{FNs} \widehat{\zeta}_{j}=$ $\left(p_{1} \circ \zeta_{j}, p_{2} \circ \zeta_{j}, \ldots, p_{m} \circ \zeta_{j}\right), \quad j=1,2, \ldots, n$, a mapping $m$ FDWG: $\widehat{\zeta}^{n} \longrightarrow \widehat{\zeta}$ is called $m$ FDWG operator, which is given by

$$
m \operatorname{FDWG}_{\Theta}\left(\widehat{\zeta}_{1}, \widehat{\zeta}_{2}, \ldots, \widehat{\zeta}_{n}\right)=\otimes_{j=1}^{n}\left(\widehat{\zeta}_{j}\right)^{\Theta_{j}}
$$

where $\Theta=\left(\Theta_{1}, \Theta_{2}, \ldots, \Theta_{n}\right)^{T}$ represents the weights, with $\sum_{j=1}^{n} \Theta_{j}=1, \Theta_{j} \in(0,1]$.

Theorem 9. For a collection of $m F N s$ $\widehat{\zeta}_{j}=\left(p_{1} \circ \zeta_{j}, \ldots, p_{m} \circ \zeta_{j}\right)$ where $j=1,2, \ldots, n$, an accumulated score of these mFNs using the mFDWG operators is defined by

$$
\begin{aligned}
\operatorname{mFDWG} \mathrm{FD}_{\Theta}\left(\widehat{\zeta}_{1}, \widehat{\zeta}_{2}, \ldots, \widehat{\zeta}_{n}\right)= & \otimes_{j=1}^{n}\left(\widehat{\zeta}_{j}\right)^{\Theta_{j}}, \\
& \left(1-\frac{1}{1+\left\{\sum_{j=1}^{n} \Theta_{j}\left(1-p_{1} \circ \zeta_{j} / p_{1} \circ \zeta_{j}\right)^{k}\right\}^{1 / k}}, \ldots, 1-\frac{1}{1+\left\{\sum_{\Theta_{j}}^{n} \Theta_{j}\left(1-p_{m} \circ \zeta_{j} / p_{m} \circ \zeta_{j}\right)^{k}\right\}^{1 / k}}\right) .
\end{aligned}
$$

Proof. Its proof is identical to Theorem 2.

Example 4. Let $\widehat{\zeta}_{1}=(0.2,0.6,0.3), \widehat{\zeta}_{2}=(0.9,1.0,0.7), \widehat{\zeta}_{3}=$ $(0.1,0.8,0.4)$, and $\widehat{\zeta}_{4}=(0.4,0.7,0.3)$ be $3 \mathrm{FNs}$ with weights $\Theta=(0.1,0.5,0.3,0.1)^{T}$. Then, for $k=3$,

$$
\begin{aligned}
& \operatorname{mFDWG}_{\Theta}\left(\widehat{\zeta}_{1}, \widehat{\zeta}_{2}, \widehat{\zeta}_{3}\right)=\otimes_{j=1}^{3}\left(\widehat{\zeta}_{j}\right)^{\Theta_{j}} \\
& =\left(1-\frac{1}{1+\left\{\sum_{j=1}^{n} \Theta_{j}\left(1-p_{1} \circ \zeta_{j} / p_{1} \circ \zeta_{j}\right)^{k}\right\}^{1 / k}}, \ldots, 1-\frac{1}{1+\left\{\sum_{j=1}^{n} \Theta_{j}\left(1-p_{m} \circ \zeta_{j} / p_{m} \circ \zeta_{j}\right)^{k}\right\}^{1 / k}}\right), \\
& =\left(1-\frac{1}{1+\left(0.1 \times(1-0.2 / 0.2)^{3}+0.5 \times(1-0.9 / 0.9)^{3}+0.3 \times(1-0.1 / 0.1)^{3}+0.1 \times(1-0.4 / 0.4)^{3}\right)^{1 / 3}}\right. \text {, } \\
& 1-\frac{1}{1+\left(0.1 \times(1-0.6 / 0.6)^{3}+0.5 \times(1-0.1 / 0.1)^{3}+0.3 \times(1-0.8 / 0.8)^{3}+0.1 \times(1-0.7 / 0.7)^{3}\right)^{1 / 3}} \text {, } \\
& \left.1-\frac{1}{1+\left(0.1 \times(1-0.3 / 0.3)^{3}+0.5 \times(1-0.7 / 0.7)^{3}+0.3 \times(1-0.4 / 0.4)^{3}+0.1 \times(1-0.3 / 0.3)^{3}\right)^{1 / 3}}\right) \text {, } \\
& =(0.8589,0.2582,0.6052) \text {. }
\end{aligned}
$$


It can be readily shown that the $m$ FDWG operator holds the notions given below.

Theorem 10 (idempotent law). Let $\widehat{\zeta}_{j}=\left(p_{1} \circ \zeta_{j}, \ldots, p_{m} \circ \zeta_{j}\right)$ be a family of " $n$ " $m F N s$, which are equal, i.e., $\widehat{\zeta}_{j}=\widehat{\zeta}_{\text {; then, }}$

$$
m \operatorname{FDWG}_{\Theta}\left(\widehat{\zeta}_{1}, \widehat{\zeta}_{2}, \ldots, \widehat{\zeta}_{n}\right)=\widehat{\zeta}
$$

Theorem 11 (bounded law). Let $\widehat{\zeta}_{j}=\left(p_{1} \circ \zeta_{j+}, \ldots, p_{m} \circ \zeta_{j}\right)$ be a collection of " $n$ " $m F N s, \widehat{\zeta}^{-}=\cap_{j=1}^{n}\left(\zeta_{j}\right)$, and $\zeta^{+}=\cup_{j=1}^{n}\left(\zeta_{j}\right)$; then,

$$
\widehat{\zeta}^{-} \leq m \operatorname{FDWG}_{\Theta}\left(\widehat{\zeta}_{1}, \widehat{\zeta}_{2}, \ldots, \widehat{\zeta}_{n}\right) \leq \widehat{\zeta}^{+}
$$

Theorem 12 (monotonic law). For two collections of $m$ FNs $\widehat{\zeta}_{j}$ and $\widehat{\zeta}_{j}^{\prime},(j=1,2, \ldots, n)$, if $\widehat{\zeta}_{j} \leq \widehat{\zeta}_{j}^{\prime}$, then

$$
m \operatorname{FDWG}_{\Theta}\left(\widehat{\zeta}_{1}, \widehat{\zeta}_{2}, \ldots, \widehat{\zeta}_{n}\right) \leq m \operatorname{FDWG}_{\Theta}\left(\hat{\zeta}_{1}^{\prime}, \hat{\zeta}_{2}^{\prime}, \ldots, \widehat{\zeta}_{n}^{\prime}\right) .
$$

Now, we develop mFDOWG operators.

Definition 9. For a family of $m \mathrm{FNs}$ $\widehat{\zeta}_{j}=\left(p_{1} \circ \zeta_{j}, p_{2} \circ \zeta_{j}, \ldots, p_{m} \circ \zeta_{j}\right), j=1,2, \ldots, n, \quad \widehat{\zeta}^{n} \quad$ an $m$ FDOWG operator is a mapping $m$ FDOWG: $\widehat{\zeta}^{n} \longrightarrow \widehat{\zeta}$, which is given as

$$
m \operatorname{FDOWG}_{w}\left(\hat{\zeta}_{1}, \widehat{\zeta}_{2}, \ldots, \widehat{\zeta}_{n}\right)=\otimes_{j=1}^{n}\left(w_{j} \widehat{\zeta}_{\sigma(j)}\right),
$$

where $w=\left(w_{1}, w_{2}, \ldots, w_{n}\right)^{T}$ is the weight vector and $w_{j} \in(0,1]$ with $\sum_{j=1}^{n} w_{j}=1 . \sigma(j), j=1,2, \ldots, n$ represents the permutation, such that $\widehat{\zeta}_{\sigma(j-1)} \geq \widehat{\zeta}_{\sigma(j)}$.

Theorem 13. For a collection of $m F N s$ $\widehat{\zeta}_{j}=\left(p_{1} \circ \zeta_{j}, \ldots, p_{m} \circ \zeta_{j}\right)$ where $j=1,2, \ldots, n$, an accumulated score of these mFNs using an mFDOWG operator is defined by

$$
\begin{aligned}
& m \operatorname{FDOWG}_{w}\left(\widehat{\zeta}_{1}, \widehat{\zeta}_{2}, \ldots, \widehat{\zeta}_{n}\right)=\otimes_{j=1}^{n}\left(\widehat{\zeta}_{\sigma(j)}\right)^{w_{j}} \\
& =\left(1-\frac{1}{1+\left\{\sum_{j=1}^{n} w_{j}\left(1-p_{1} \circ \zeta_{\sigma(j)} / p_{1} \circ \zeta_{\sigma(j)}\right)^{k}\right\}^{1 / k}}, \ldots, 1-\frac{1}{1+\left\{\sum_{j=1}^{n} w_{j}\left(1-p_{m} \circ \zeta_{\sigma(j)} / p_{m} \circ \zeta_{\sigma(j)}\right)^{k}\right\}^{1 / k}}\right) .
\end{aligned}
$$

Example 5. Let $\widehat{\zeta}_{1}=(0.2,0.4,0.7), \widehat{\zeta}_{2}=(0.3,0.6,0.1), \widehat{\zeta}_{3}=$ $(0.8,0.3,0.5)$, and $\widehat{\zeta}_{4}=(0.6,0.4,0.7)$ be $3 \mathrm{FNs}$ and $w=(0.3,0.1,0.2,0.4)^{T}$ be a weight vector. Then, score values of $m \mathrm{FNs}$ for $k=3$ are calculated as

$$
\begin{aligned}
& S\left(\widehat{\zeta}_{1}\right)=\frac{0.2+0.4+0.7}{3}=0.4333 \\
& S\left(\widehat{\zeta}_{2}\right)=\frac{0.3+0.6+0.1}{3}=0.3333, \\
& S\left(\widehat{\zeta}_{3}\right)=\frac{0.8+0.3+0.5}{3}=0.5333, \\
& S\left(\widehat{\zeta}_{4}\right)=\frac{0.6+0.4+0.7}{3}=0.5667 .
\end{aligned}
$$

$$
\begin{aligned}
\text { Since } S\left(\widehat{\zeta}_{4}\right)> & S\left(\widehat{\zeta}_{3}\right)>S\left(\widehat{\zeta}_{1}\right)>S\left(\widehat{\zeta}_{2}\right), \\
\widehat{\zeta}_{\sigma(1)} & =\widehat{\zeta}_{3}=(0.6,0.4,0.7), \\
\widehat{\zeta}_{\sigma(2)} & =\widehat{\zeta}_{3}=(0.8,0.3,0.5), \\
\widehat{\zeta}_{\sigma(3)} & =\widehat{\zeta}_{1}=(0.2,0.4,0.7), \\
\widehat{\zeta}_{\sigma(4)} & =\widehat{\zeta}_{2}=(0.3,0.6,0.1) .
\end{aligned}
$$

Then, from Definition 9, 


$$
\begin{aligned}
& m \operatorname{FDOWG}_{w}\left(\widehat{\zeta}_{1}, \widehat{\zeta}_{2}, \widehat{\zeta}_{3}, \widehat{\zeta}_{4}\right)=\otimes_{j=1}^{4}\left(\widehat{\zeta}_{\sigma(j)}\right)^{w_{j}} \\
& =\left(1-\frac{1}{1+\left\{\sum_{j=1}^{4} w_{j}\left(1-p_{1} \circ \zeta_{\sigma(j)} / p_{1} \circ \zeta_{\sigma(j)}\right)^{k}\right\}^{1 / k}}, \ldots, 1-\frac{1}{1+\left\{\sum_{j=1}^{4} w_{j}\left(1-p_{3} \circ \zeta_{\sigma(j)} / p_{3} \circ \zeta_{\sigma(j)}\right)^{k}\right\}^{1 / k}}\right), \\
& =\left(1-\frac{1}{1+\left(0.3 \times(1-0.6 / 0.6)^{3}+0.1 \times(1-0.8 / 0.8)^{3}+0.2 \times(1-0.2 / 0.2)^{3}+0.4 \times(1-0.3 / 0.3)^{3}\right)^{1 / 3}},\right. \\
& 1-\frac{1}{1+\left(0.3 \times(1-0.4 / 0.4)^{3}+0.1 \times(1-0.3 / 0.3)^{3}+0.2 \times(1-0.4 / 0.4)^{3}+0.4 \times(1-0.6 / 0.6)^{3}\right)^{1 / 3}}, \\
& \left.1-\frac{1}{1+\left(0.3 \times(1-0.7 / 0.7)^{3}+0.1 \times(1-0.5 / 0.5)^{3}+0.2 \times(1-0.7 / 0.7)^{3}+0.4 \times(1-0.1 / 0.1)^{3}\right)^{1 / 3}}\right), \\
& =(0.7237,0.5926,0.8690) \text {. }
\end{aligned}
$$

Remark 2. Note that $m$ FDOWG operators satisfy properties, namely, idempotency, boundedness, and monotonicity, as described in Theorems 10, 11, and 12 .

Theorem 14 (commutative law). For two arbitrary collections of $\operatorname{mFNs} \widehat{\zeta}_{j}$ and $\widehat{\zeta}_{j}^{\prime}(j=1,2, \ldots, n)$, if $\widehat{\zeta}_{j} \leq \widehat{\zeta}_{j}^{\prime}$, then

$$
m \operatorname{FDOWG}_{w}\left(\widehat{\zeta}_{1}, \widehat{\zeta}_{2}, \ldots, \widehat{\zeta}_{n}\right)=m \operatorname{FDOWG}_{w}\left(\widehat{\zeta}_{1}^{\prime}, \widehat{\zeta}_{2}^{\prime}, \ldots, \widehat{\zeta}_{n}^{\prime}\right) \text {, }
$$

where $\widehat{\zeta}_{j}^{\prime}$ is any permutation of $\hat{\zeta}_{j}$.

In Definitions 5 and 6 , we see that $m$ FDWG and $m$ FDOWG operators aggregate weighted $m$ FNs and their ordering, respectively. Now, we develop a new operator called $m$ FDHG operator, which contains the properties of both $m$ FDWG and $m$ FDOWG operators.

Definition 10. For a family of $m \mathrm{FNs}$ $\widehat{\zeta}_{j}=\left(p_{1} \circ \zeta_{j}, p_{2} \circ \zeta_{j}, \ldots, p_{m} \circ \zeta_{j}\right), j=1,2, \ldots, n$, an $m$ FDHG operator is defined by

$$
\begin{aligned}
\operatorname{mDDHG}_{w, \Theta}\left(\widehat{\zeta}_{1}, \widehat{\zeta}_{2}, \ldots, \widehat{\zeta}_{n}\right) & =\otimes_{j=1}^{n}\left(\widetilde{\widetilde{\zeta}}_{\sigma(j)}\right)^{w_{j}}, \\
& =\left(1-\frac{1}{\left.1+\left\{\sum_{j=1}^{n} w_{j}\left(1-p_{1} \circ \zeta_{\sigma(j)} / p_{1} \circ \zeta_{\sigma(j)}\right)^{k}\right\}^{1 / k}, \ldots, 1-\frac{1}{1+\left\{\sum_{j=1}^{n} w_{j}\left(1-p_{m} \circ \zeta_{\sigma(j)} / p_{m} \circ \zeta_{\sigma(j)}\right)^{k}\right\}^{1 / k}}\right)} .\right.
\end{aligned}
$$

$$
m \operatorname{FDHG}_{w, \Theta}\left(\widehat{\zeta}_{1}, \widehat{\zeta}_{2}, \ldots, \widehat{\zeta}_{n}\right)=\otimes_{j=1}^{n}\left(\widetilde{\widetilde{\zeta}}_{\sigma(j)}\right)^{w_{j}}
$$

where $w=\left(w_{1}, w_{2}, \ldots, w_{n}\right)^{T}$ denotes the weights associated to the $m$ FNs $\widehat{\zeta}_{j}, j=1,2, \ldots, n, w_{j} \in(0,1], \sum_{j=1}^{n} w_{j}=1$, $\zeta_{\sigma(j)}=\left(n \Theta_{j}\right) \widehat{\zeta}_{j},(j=1,2, \ldots, n)$, and $\Theta=\left(\Theta_{1}, \Theta_{2}, \ldots, \Theta_{n}\right)$ is a vector having weights, with $\Theta_{j} \in(0,1], \sum_{j=1}^{n} \Theta_{j}=1$.

Notice that when $w=((1 / n),(1 / n), \ldots,(1 / n))^{T}$, $m$ FDHG operator becomes $m$ FDWG operator. When $\Theta=((1 / n),(1 / n), \ldots,(1 / n))^{T}$, then $m$ FDHG operator converts into $m$ FDOWG operator. Thus, $m$ FDHG operator is a generalization of $m$ FDWG and $m$ FDOWG operators.

With the induction technique, one can readily show the next theorem.

Theorem 15. For a collection of mFNs $\widehat{\zeta}_{j}=\left(p_{1} \circ \zeta_{j}, \ldots, p_{m} \circ \zeta_{j}\right)$ where $j=1,2, \ldots, n$, an accumulated score of these mFNs using an mFDHG operator is defined as $\widehat{\zeta}_{z \sigma(j)} \quad$ is the $j$-th largest $m \mathrm{FN}$, 
Example 6. Let $\widehat{\zeta}_{1}=(0.4,0.6,0.3), \widehat{\zeta}_{2}=(0.3,0.2,0.9)$, $\widehat{\zeta}_{3}=(0.6,0.3,0.5)$, and $\widehat{\zeta}_{4}=(0.3,0.5,0.7)$ be 3 FNs and $w=$ $(0.4,0.1,0.3,0.2)^{T}$ be an associated weight vector and a vector $\Theta=(0.5,0.2,0.1,0.2)^{T}$ be weights. By Definition 10, for $k=3$,

$$
\begin{aligned}
\widetilde{\widetilde{\zeta}}_{1} & =\left(1-\frac{1}{1+\left\{n \Theta_{1}\left(1-p_{1} \circ \zeta_{1} / p_{1} \circ \zeta_{1}\right)^{k}\right\}^{1 / k}}, \ldots, 1-\frac{1}{1+\left\{n \Theta_{1}\left(1-p_{3} \circ \zeta_{1} / p_{3} \circ \zeta_{1}\right)^{k}\right\}^{1 / k}}\right) \\
& =\left(1-\frac{1}{1+\left(4 \times 0.5 \times(1-0.4 / 0.4)^{3}\right)^{1 / 3}}, 1-\frac{1}{1+\left(4 \times 0.5 \times(1-0.6 / 0.6)^{3}\right)^{1 / 3}}, 1-\frac{1}{1+\left(4 \times 0.5 \times(1-0.3 / 0.3)^{3}\right)^{1 / 3}}\right) \\
& =(0.6540,0.4565,0.7462) .
\end{aligned}
$$

Similarly,

$$
\begin{aligned}
\widetilde{\widehat{\zeta}}_{2} & =\left(1-\frac{1}{1+\left(4 \times 0.2 \times(1-0.3 / 0.3)^{3}\right)^{1 / 3}}, 1-\frac{1}{1+\left(4 \times 0.2 \times(1-0.2 / 0.2)^{3}\right)^{1 / 3}}, 1-\frac{1}{1+\left(4 \times 0.2 \times(1-0.9 / 0.9)^{3}\right)^{1 / 3}}\right) \\
& =(0.6842,0.7878,0.0935),
\end{aligned}
$$

$$
\begin{aligned}
\widetilde{\widetilde{\zeta}}_{3} & =\left(1-\frac{1}{1+\left(4 \times 0.1 \times(1-0.6 / 0.6)^{3}\right)^{1 / 3}}, 1-\frac{1}{1+\left(4 \times 0.1 \times(1-0.3 / 0.3)^{3}\right)^{1 / 3}}, 1-\frac{1}{1+\left(4 \times 0.1 \times(1-0.5 / 0.5)^{3}\right)^{1 / 3}}\right) \\
& =(0.3294,0.6322,0.4242),
\end{aligned}
$$

$$
\begin{aligned}
\widetilde{\widetilde{\zeta}}_{4} & =\left(1-\frac{1}{1+\left(4 \times 0.2 \times(1-0.3 / 0.3)^{3}\right)^{1 / 3}}, 1-\frac{1}{1+\left(4 \times 0.2 \times(1-0.5 / 0.5)^{3}\right)^{1 / 3}}, 1-\frac{1}{1+\left(4 \times 0.2 \times(1-0.7 / 0.7)^{3}\right)^{1 / 3}}\right) \\
& =(0.6842,0.4814,0.2846)
\end{aligned}
$$

Then, score values of $m$ FNs for $k=3$ are given as follows:

$$
\begin{aligned}
& S\left(\widetilde{\zeta}_{1}\right)=\frac{0.6540+0.4565+0.7462}{3}=0.6189, \\
& S\left(\widetilde{\zeta}_{2}\right)=\frac{0.6842+0.7878+0.0935}{3}=0.5218, \\
& S\left(\widetilde{\zeta}_{3}\right)=\frac{0.3294+0.6322+0.4242}{3}=0.4620, \\
& S\left(\widetilde{\widetilde{\zeta}}_{4}\right)=\frac{0.6842+0.4814+0.2846}{3}=0.4834 .
\end{aligned}
$$

$$
\text { Since } S\left(\widetilde{\widetilde{\zeta}}_{1}\right)>S\left(\widetilde{\widetilde{\zeta}}_{2}\right)>S\left(\widetilde{\widetilde{\zeta}}_{4}\right)>S\left(\widetilde{\widetilde{\zeta}}_{3}\right)
$$

$$
\begin{aligned}
& \widetilde{\widehat{\zeta}}_{\sigma(1)}=\widehat{\zeta}_{1}=(0.6540,0.4565,0.7462) \\
& \widetilde{\widetilde{\zeta}}_{\sigma(2)}=\widehat{\zeta}_{2}=(0.6842,0.7878,0.0935) \\
& \widetilde{\zeta}_{\sigma(3)}=\widehat{\zeta}_{4}=(0.6842,0.4814,0.2846) \\
& \widetilde{\widetilde{\zeta}}_{\sigma(4)}=\widehat{\zeta}_{3}=(0.3294,0.6322,0.4242)
\end{aligned}
$$

Then, from Definition 9, 


$$
\begin{aligned}
m \mathrm{FDH} \zeta_{w, \Theta}\left(\widehat{\zeta}_{1}, \widehat{\zeta}_{2}, \widehat{\zeta}_{3}, \widehat{\zeta}_{4}\right)= & \otimes_{j=1}^{4}\left(\widehat{\zeta}_{\sigma(j)}\right)^{w_{j}}, \\
= & \left(1-\frac{1}{1+\left\{\sum_{j=1}^{4} w_{j}\left(1-p_{1} \circ \zeta_{\sigma(j)} / p_{1} \circ \zeta_{\sigma(j)}\right)^{k}\right\}^{1 / k}}, \ldots, 1-\frac{1}{1+\left\{\sum_{j=1}^{4} w_{j}\left(1-p_{3} \circ \zeta_{\sigma(j)} / p_{3} \circ \zeta_{\sigma(j)}\right)^{k}\right\}}\right) \\
= & \left(1-\frac{1}{1+\left(0.4 \times(1-0.6540 / 0.6540)^{3}+0.1 \times(1-0.6842 / 0.6842)^{3}+0.3 \times(1-0.6842 / 0.6842)^{3}+0.2 \times(1-0.3294 / 0.3294)^{3}\right)^{1 / 3}},\right. \\
& 1-\frac{1}{1+\left(0.4 \times(1-0.4565 / 0.4565)^{3}+0.1 \times(1-0.7878 / 0.7878)^{3}+0.3 \times(1-0.4814 / 0.4814)^{3}+0.2 \times(1-0.6322 / 0.6322)^{3}\right)^{1 / 3},} \\
& \left.1-\frac{1}{1+\left(0.4 \times(1-0.7462 / 0.7462)^{3}+0.1 \times(1-0.0935 / 0.0935)^{3}+0.3 \times(1-0.2846 / 0.2846)^{3}+0.2 \times(1-0.4242 / 0.4242)^{3}\right)^{1 / 3}}\right), \\
= & (0.5482,0.5073,0.8210) .
\end{aligned}
$$

\section{Mathematical Method for MCDM with $m$ F Data}

To solve MCDM problems containing $m \mathrm{~F}$ data, we apply $m \mathrm{~F}$ Dombi AOs. The following notions are utilized to tackle the MCDM situations having $m \mathrm{~F}$ information. Suppose that $\left\{Y_{1}, Y_{2}, \ldots, Y_{k}\right\}$ is a universal set and $\left\{S_{1}, S_{2}, \ldots, S_{n}\right\}$ is the universe of attributes. Assume $\Theta=\left\{\Theta_{1}, \Theta_{2}, \ldots, \Theta_{n}\right\}$ is a weight vector with $\sum_{j=1}^{n} \Theta_{j}=1, \Theta_{j} \in(0,1]$, for all $j=1, \ldots, n$. Consider $\widehat{S}=\left(\widehat{s}_{i j}\right)_{k \times n}=\left(p_{1} \circ \zeta_{i j}, p_{2} \circ \zeta_{i j}, \ldots, p_{m} \circ\right.$ $\left.\zeta_{i j}\right)_{k \times n}$ is an $m \mathrm{~F}$ decision matrix, which represents the membership values evaluated by the experts.

We construct an algorithmic method to handle MCDM problems by an $m$ FDWA (or $m$ FDWG) operator.

\section{Applications}

5.1. Agriculture Land Selection. Agriculture is an essential part of Pakistan's economic system. This area directly supports the population of the country and accounts for $26 \%$ of gross domestic product (GDP). The leading agricultural crops include sugarcane, wheat, rice, cotton, vegetables, and fruits. A business man wants to invest in agriculture sector and is searching for an appropriate land. The options in his brain are $Y_{1}, Y_{2}, \ldots, Y_{5}$. He consults an expert to get his suggestion about the alternatives based on the following desired parameters:

$$
\begin{aligned}
& S_{1} \text { denotes the "Location" } \\
& S_{2} \text { denotes the "Climate" } \\
& S_{3} \text { denotes the "Fertility" } \\
& S_{4} \text { denotes the "Price" }
\end{aligned}
$$

Each parameter has been characterized into three parts to construct a $3 \mathrm{FN}$.

(i) "Location" includes near to market, near to water channel, and transport availability.

(ii) "Climate" includes temperature, pollution level, and humidity level.

(iii) "Fertility" includes soil PH, level of nutrients, and water retention capacity of land. (iv) "Price" includes low, medium, and high.

The $3 \mathrm{~F}$ decision matrix is shown in Table 1 .

According to the businessman, the expert assigns weights to parameters as follows:

$$
\begin{aligned}
& \Theta_{1}=0.35, \\
& \Theta_{2}=0.25, \\
& \Theta_{3}=0.30, \\
& \Theta_{4}=0.10 .
\end{aligned}
$$

Clearly, $\sum_{j=1}^{3} \Theta_{j}=1$. To compute the most suitable land regarding agriculture, we use the two operators, namely, $m$ FDWA and $m$ FDWG, respectively:

(1) For $k=3$, by applying the mFDWA operator, we calculate the values $\widehat{s}_{i}$ of the lands $Y_{i}, i=1,2, \ldots, 5$ regarding agriculture.

$$
\begin{aligned}
& \widehat{s}_{1}=(0.8107,0.6224,0.6109), \\
& \widehat{s}_{2}=(0.8662,0.6679,0.7297), \\
& \widehat{s}_{3}=(0.5443,0.7418,0.6515), \\
& \widehat{s}_{4}=(0.8192,0.7347,0.5366), \\
& \widehat{s}_{5}=(0.8663,0.6334,0.6327) .
\end{aligned}
$$

(2) Find the score values $S\left(\widehat{s}_{i}\right)$ of $3 \mathrm{FNs}$ $\widehat{s}_{i},(i=1,2, \ldots, 5)$ of the lands $Y_{i}$ :

$$
\begin{aligned}
& S\left(\widehat{s}_{1}\right)=0.6814, \\
& S\left(\widehat{s}_{2}\right)=0.7546, \\
& S\left(\widehat{s}_{3}\right)=0.6459, \\
& S\left(\widehat{s}_{4}\right)=0.6968, \\
& S\left(\widehat{s}_{5}\right)=0.7108 .
\end{aligned}
$$

(3) Rank the lands using scores $S\left(s_{i}\right),(i=1,2, \ldots, 5)$ obtained from the preference values in the form of 3FNs: $Y_{2}>Y_{5}>Y_{4}>Y_{1}>Y_{3}$. 
TABLE 1: 3F decision matrix.

\begin{tabular}{ccccc}
\hline & $S_{1}$ & $S_{2}$ & $S_{3}$ & $S_{4}$ \\
\hline$Y_{1}$ & $(0.7,0.6,0.3)$ & $(0.5,0.7,0.2)$ & $(0.6,0.3,0.7)$ & $(0.9,0.5,0.4)$ \\
$Y_{2}$ & $(0.9,0.6,0.5)$ & $(0.8,0.5,0.3)$ & $(0.4,0.5,0.8)$ & $(0.5,0.8,0.5)$ \\
$Y_{3}$ & $(0.4,0.7,0.3)$ & $(0.5,0.4,0.3)$ & $(0.6,0.8,0.4)$ & $(0.6,0.3,0.8)$ \\
$Y_{4}$ & $(0.5,0.6,0.3)$ & $(0.8,0.6,0.5)$ & $(0.7,0.8,0.2)$ & $(0.9,0.3,0.7)$ \\
$Y_{5}$ & $(0.9,0.7,0.6)$ & $(0.8,0.4,0.3)$ & $(0.6,0.5,0.7)$ & $(0.4,0.6,0.5)$ \\
\hline
\end{tabular}

(4) $Y_{2}$ has a high score value, so it is the best land for agriculture.

In a similar way, apply an $m$ FDWG operator to find an appropriate land.

(1) Take $k=3$. Apply an $m$ FDWG operator to determine the values $\widehat{s}_{i}$ of the lands $Y_{i}$.

$$
\begin{aligned}
& \widehat{s}_{1}=(0.4171,0.6142,0.7334), \\
& \widehat{s}_{2}=(0.5092,0.4648,0.6058), \\
& \widehat{s}_{3}=(0.5364,0.5633,0.6723), \\
& \widehat{s}_{4}=(0.4196,0.5311,0.7423), \\
& \widehat{s}_{5}=(0.4303,0.5153,0.6007) .
\end{aligned}
$$

(2) Determine the score values $S\left(\widehat{s}_{i}\right)$ of $3 \mathrm{FNs} \widehat{s}_{i}$ of the lands $Y_{i}$ :

$$
\begin{aligned}
& S\left(\widehat{s}_{1}\right)=0.5882, \\
& S\left(\widehat{s}_{2}\right)=0.5266, \\
& S\left(\widehat{s}_{3}\right)=0.5907, \\
& S\left(\widehat{s}_{4}\right)=0.5643, \\
& S\left(\widehat{s}_{5}\right)=0.5154 .
\end{aligned}
$$

(3) Rank the lands using scores $S\left(\widehat{s}_{i}\right),(i=1,2, \ldots, 5)$ obtained from the preference values in the form of 3FNs: $Y_{3}>Y_{1}>Y_{4}>Y_{2}>Y_{5}$.

(4) $Y_{3}$ has high score, so it is the best land for agriculture.

5.2. Performance Evaluation of Commercial Banks. Commercial bank is one of the largest essential economic institutions. It can pull in money related streams, offering credit and different monetary administrations. These activities vitally affect national monetary improvements. Hence, commercial banks ought to be assessed by the modern and reliable procedures to rank commercial banks in the financial framework. This research establishes a MCDM model that uses $m$ FDWA, $m$ FDWG, and $m$ F ELECTRE-I methods under a set of criteria and rank commercial banks. The board of specialists will assess each bank under chosen criteria. After a primer evaluation, six banks $\left\{B_{1}, B_{2}, B_{3}, B_{4}, B_{5}, B_{6}\right\}$ are assessed and ranked to pick the best bank. The banks are evaluated on the basis of four parameters.

$$
\begin{aligned}
& S_{1} \text { denotes the "Net Income" } \\
& S_{2} \text { denotes the "Customer Service" }
\end{aligned}
$$

$S_{3}$ denotes the "Nonfinancial Performance"

$S_{4}$ denotes the "Potential Attractiveness"

Each parameter has been characterized into four parts to form a $4 \mathrm{FN}$.

(i) "Net Income" includes total equity, operating income, total assets, and net interest income.

(ii) "Customer Service" includes accessibility for customers, the evaluation of Internet page, the number of new services, and the number of new products.

(iii) "Nonfinancial Performance" includes support from main stake holders, bank management, employee stability, and ownership structure.

(iv) "Potential Attractiveness" includes location, involving environment, strategic dimension, and external and internal characteristics.

The $4 \mathrm{~F}$ decision matrix is represented by Table 2 .

The expert assigns weights to parameters as follows:

$$
\begin{aligned}
& \Theta_{1}=0.28, \\
& \Theta_{2}=0.34, \\
& \Theta_{3}=0.22, \\
& \Theta_{4}=0.16 .
\end{aligned}
$$

Clearly, $\sum_{j=1}^{4} \Theta_{j}=1$. To select the most efficient bank, we use the two operators, namely, $m$ FDWA and $m$ FDWG, respectively:

(1) For $k=3$, utilize the $m$ FDWA operator to compute the values $\widehat{s}_{i}$ for the banks $B_{i}, i=1,2, \ldots, 6$.

$$
\begin{aligned}
& \widehat{s}_{1}=(0.6188,0.5392,0.6306,0.6723), \\
& \widehat{s}_{2}=(0.8617,0.8639,0.7895,0.6875), \\
& \widehat{s}_{3}=(0.5139,0.7319,0.5492,0.5097), \\
& \widehat{s}_{4}=(0.7385,0.6188,0.5699,0.5492), \\
& \widehat{s}_{5}=(0.6551,0.6323,0.5227,0.6112), \\
& \widehat{s}_{6}=(0.7689,0.7302,0.6562,0.6228) .
\end{aligned}
$$

(2) Calculate the score values $S\left(\widehat{s}_{i}\right)$ of $4 \mathrm{FNs}$ $\widehat{s}_{i},(i=1,2, \ldots, 6)$ for the banks $B_{i}$.

$$
\begin{aligned}
& S\left(\widehat{s}_{1}\right)=0.6152, \\
& S\left(\widehat{s}_{2}\right)=0.8007, \\
& S\left(\widehat{s}_{3}\right)=0.5762, \\
& S\left(\widehat{s}_{4}\right)=0.6191, \\
& S\left(\widehat{s}_{5}\right)=0.6053, \\
& S\left(\widehat{s}_{6}\right)=0.6945 .
\end{aligned}
$$

(3) Now, rank the banks using scores $S\left(s_{i}\right),(i=1,2, \ldots, 6)$ obtained from the preference values in the form of $4 \mathrm{~F}$ numbers: $B_{2}>B_{6}>B_{4}>B_{1}>B_{5}>B_{3}$.

(4) $B_{2}$ has a high score value, so it is the best bank. 
TABLE 2: $4 \mathrm{~F}$ decision matrix.

\begin{tabular}{lccrr}
\hline & $S_{1}$ & $S_{2}$ & $S_{3}$ & $S_{4}$ \\
\hline$B_{1}$ & $(0.7,0.6,0.7,0.7)$ & $(0.5,0.7,0.6,0.7)$ & $(0.5,0.3,0.5,0.5)$ & $(0.5,0.4,0.4,0.6)$ \\
$B_{2}$ & $(0.9,0.7,0.8,0.7)$ & $(0.8,0.9,0.8,0.7)$ & $(0.8,0.7,0.7,0.6)$ & $(0.7,0.7,0.8,0.7)$ \\
$B_{3}$ & $(0.4,0.8,0.5,0.6)$ & $(0.5,0.6,0.6,0.4)$ & $(0.5,0.6,0.50 .4)$ & $(0.6,0.6,0.5,0.3)$ \\
$B_{4}$ & $(0.8,0.7,0.6,0.5)$ & $(0.7,0.5,0.4,0.6)$ & $(0.5,0.5,0.6,0.5)$ & $(0.4,0.5,0.6,0.5)$ \\
$B_{5}$ & $(0.7,0.7,0.6,0.7)$ & $(0.5,0.6,0.4,0.4)$ & $(0.7,0.5,0.5,0.4)$ & $(0.5,0.5,0.4,0.5)$ \\
$B_{6}$ & $(0.8,0.8,0.7,0.6)$ & $(0.7,0.6,0.6,0.5)$ & $(0.8,0.5,0.5,0.4)$ & $(0.6,0.6,0.7,0.6)$ \\
\hline
\end{tabular}

In a similar way, apply the $m$ FDWG operator to determine the most efficient bank.

(1) Take $k=3$. We employ the $m$ FDWG operator to compute the values $\widehat{s}_{i}$ for the banks $B_{i}$.

$$
\begin{aligned}
& \widehat{s}_{1}=(0.4752,0.6016,0.4896,0.4052), \\
& \widehat{s}_{2}=(0.2182,0.2723,0.2361,0.3343), \\
& \widehat{s}_{3}=(0.5366,0.3756,0.4772,0.6136), \\
& \widehat{s}_{4}=(0.4006,0.5206,0.5246,0.4772), \\
& \widehat{s}_{5}=(0.4487,0.4429,0.4952,0.5604), \\
& \widehat{s}_{6}=(0.3029,0.4185,0.4146,0.5118)
\end{aligned}
$$

(2) Find the score values $S\left(\widehat{s}_{i}\right)$ of $4 \mathrm{FNs} \widehat{s}_{i}$ for the banks $B_{i}$.

$$
\begin{aligned}
& S\left(\widehat{s}_{1}\right)=0.4929, \\
& S\left(\widehat{s}_{2}\right)=0.2652, \\
& S\left(\widehat{s}_{3}\right)=0.5008, \\
& S\left(\widehat{s}_{4}\right)=0.4808, \\
& S\left(\widehat{s}_{5}\right)=0.4868, \\
& S\left(\widehat{s}_{6}\right)=0.4269 .
\end{aligned}
$$

(3) Rank the banks with scores $S\left(\widehat{s}_{i}\right),(i=1,2, \ldots, 6)$ obtained from the preference values in the form of $4 \mathrm{~F}$ numbers: $B_{3}>B_{1}>B_{5}>B_{4}>B_{6}>B_{2}$.

(4) $B_{3}$ has high score, so it is the best bank.

The methodology utilized in the applications to find the best alternative is shown in Figure 1.

5.3. $m$ F-ELECTRE-I Method. In this section, we apply $m$ F-ELECTRE-I approach [27] to the problem (performance evaluation of commercial banks, Section 5.2) (Tables 3-5).

(1) Table 3 describes the $4 \mathrm{~F}$ decision matrix.

(2) Tables 4 and 5, respectively, describe the $4 \mathrm{~F}$ concordance and discordance values.

(3) The $4 \mathrm{~F}$ concordance matrix is calculated by

$$
F=\left(\begin{array}{cccccc}
- & 0 & 0.84 & 0.62 & 0.62 & 0.34 \\
1 & - & 1 & 1 & 1 & 1 \\
0.16 & 0 & - & 0.16 & 0.34 & 0 \\
0.38 & 0 & 1 & - & 0.56 & 0.22 \\
0.66 & 0 & 0.66 & 0.44 & - & 0 \\
0.66 & 0 & 1 & 0.78 & 1 & -
\end{array}\right) .
$$

(4) The 4F concordance level $\bar{f}=0.498$.

(5) The $4 \mathrm{~F}$ discordance matrix is computed by

$G=\left(\begin{array}{cccccc}- & 1 & 0.522 & 0.289 & 0.7484 & 1 \\ 0 & - & 0 & 0 & 0 & 0 \\ 1 & 1 & - & 1 & 1 & 1 \\ 1 & 1 & 0.413 & - & 1 & 0.8303 \\ 1 & 1 & 0.7010 & 1 & - & 1 \\ 0.95 & 0 & 0 & 1 & 0 & -\end{array}\right)$.

(6) The 4F discordance level $\bar{g}=0.65$.

(7) The $4 \mathrm{~F}$ concordance and discordance matrices are given by

$$
\begin{aligned}
H & =\left(\begin{array}{cccccc}
- & 0 & 0 & 1 & 1 & 0 \\
1 & - & 1 & 1 & 1 & 1 \\
0 & 0 & - & 0 & 0 & 0 \\
0 & 0 & 1 & - & 1 & 0 \\
1 & 0 & 1 & 0 & - & 0 \\
1 & 0 & 1 & 1 & 1 & -
\end{array}\right), \\
L & =\left(\begin{array}{cccccc}
- & 0 & 1 & 1 & 0 & 0 \\
0 & - & 1 & 1 & 1 & 1 \\
0 & 0 & - & 0 & 0 & 0 \\
0 & 0 & 1 & - & 0 & 0 \\
0 & 0 & 0 & 0 & - & 0 \\
0 & 1 & 1 & 0 & 1 & -
\end{array}\right) .
\end{aligned}
$$

(8) The 4F aggregated dominance matrix is constructed as 


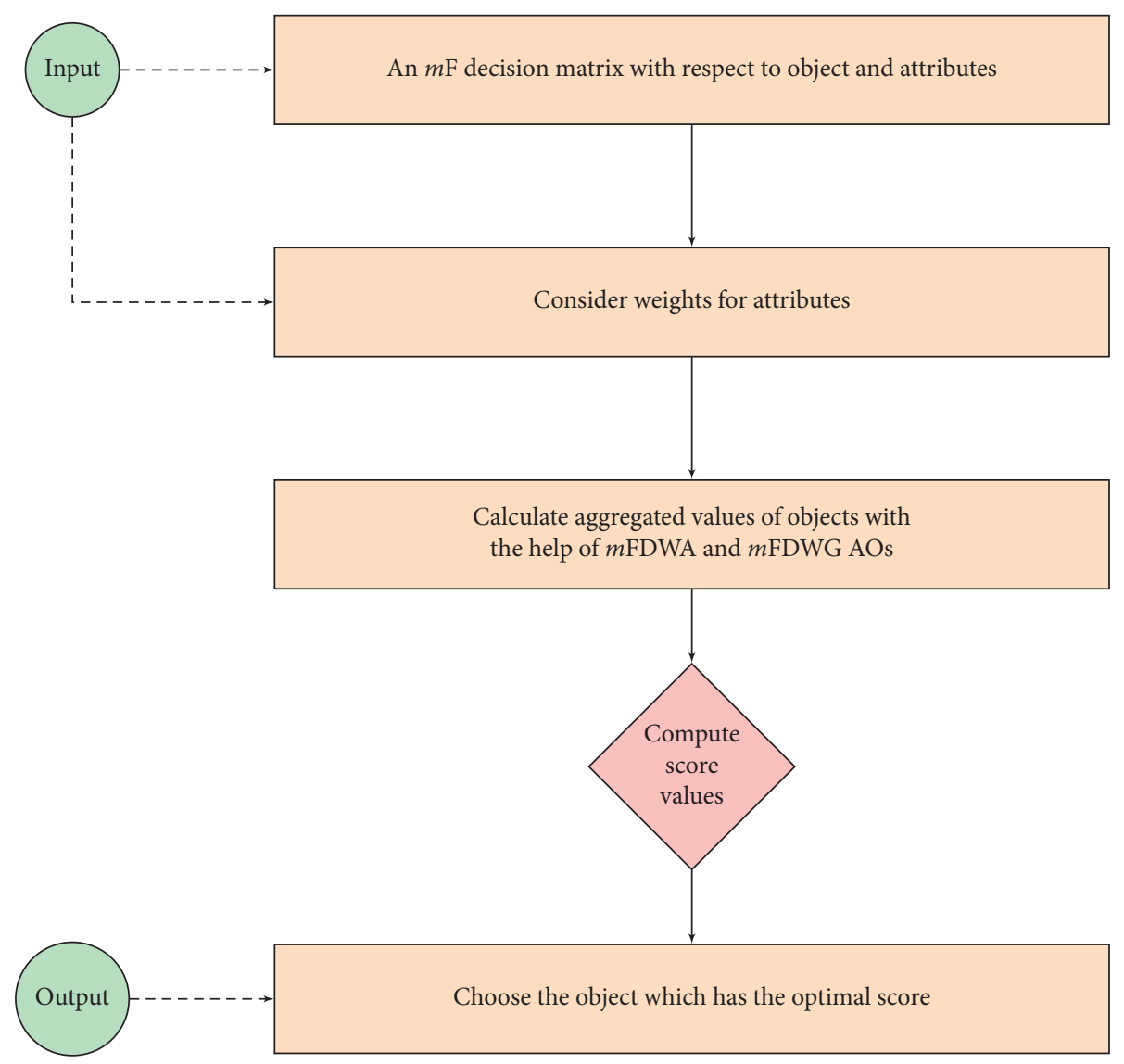

FIGURE 1: Flowchart of selecting the best option.

TABle 3: 4F weighted decision matrix.

\begin{tabular}{ccccc}
\hline & $S_{1}$ & $S_{2}$ & $S_{3}$ & $S_{4}$ \\
\hline$B_{1}$ & $(0.196,0.168,0.196,0.196)$ & $(0.17,0.238,0.204,0.238)$ & $(0.11,0.066,0.11,0.11)$ & $(0.08,0.064,0.064,0.096)$ \\
$B_{2}$ & $(0.252,0.196,0.224,0.196)$ & $(0.272,0.306,0.272,0.238)$ & $(0.176,0.154,0.154,0.132)$ & $(0.112,0.112,0.128,0.112)$ \\
$B_{3}$ & $(0.112,0.224,0.14,0.168)$ & $(0.17,0.204,0.204,0.136)$ & $(0.11,0.132,0.11,0.038)$ & $(0.096,0.096,0.08,0.048)$ \\
$B_{4}$ & $(0.224,0.196,0.168,0.14)$ & $(0.238,0.17,0.136,0.204)$ & $(0.11,0.11,0.132,0.11)$ & $(0.064,0.08,0.096,0.08)$ \\
$B_{5}$ & $(0.196,0.196,0.168,0.196)$ & $(0.17,0.204,0.136,0.136)$ & $(0.154,0.11,0.11,0.038)$ & $(0.08,0.08,0.096,0.08)$ \\
$B_{6}$ & $(0.224,0.224,0.196,0.168)$ & $(0.238,0.204,0.204,0.17)$ & $(0.176,0.11,0.11,0.038)$ & $(0.096,0.096,0.112,0.096)$ \\
\hline
\end{tabular}

TABLE 4: 4F concordance set.

\begin{tabular}{lcccccc}
\hline$j$ & 1 & 2 & 3 & 4 & 5 & 6 \\
\hline$F_{1 j}$ & - & \{\} & $\{1,2,3\}$ & $\{1,2\}$ & $\{1,2\}$ & $\{2\}$ \\
$F_{2 j}$ & $\{1,2,3,4\}$ & - & $\{1,2,3,4\}$ & $\{1,2,3,4\}$ & $\{1,2,3,4\}$ & $\{1,2,3,4\}$ \\
$F_{3 j}$ & $\{4\}$ & \{\} & - & $\{4\}$ & $\{2\}$ & \{\} \\
$F_{4 j}$ & $\{3,4\}$ & \{\} & $\{1,2,3,4\}$ & - & $\{2,3\}$ & $\{3\}$ \\
$F_{5 j}$ & $\{1,3,4\}$ & \{\} & $\{1,3,4\}$ & $\{1,4\}$ & - & \{\} \\
$F_{6 j}$ & $\{1,3,4\}$ & \{\} & $\{1,2,3,4\}$ & $\{1,2,4\}$ & $\{1,2,3,4\}$ & - \\
\hline
\end{tabular}

TABLe 5: 4F discordance set.

\begin{tabular}{lcccccc}
\hline$j$ & 1 & 2 & 3 & 4 & 5 & 6 \\
\hline$G_{1 j}$ & - & $\{1,2,3,4\}$ & $\{4\}$ & $\{3,4\}$ & $\{1,3,4\}$ & $\{1,3,4\}$ \\
$G_{2 j}$ & \{\} & - & \{\} & \{\} & \{\} & \{\} \\
$G_{3 j}$ & $\{1,2,3\}$ & $\{1,2,3,4\}$ & - & $\{1,2,3,4\}$ & $\{1,3,4\}$ & $\{1,2,3,4\}$ \\
$G_{4 j}$ & $\{1,2\}$ & $\{1,2,3,4\}$ & $\{4\}$ & - & $\{1,4\}$ & $\{1,2,4\}$ \\
$G_{5 j}$ & $\{1,2\}$ & $\{1,2,3,4\}$ & $\{2\}$ & $\{2,3\}$ & - & $\{1,2,3,4\}$ \\
$G_{6 j}$ & $\{2\}$ & \{\} & \{\} & $\{3\}$ & \{\} & - \\
\hline
\end{tabular}




$$
M=\left(\begin{array}{cccccc}
- & 0 & 0 & 1 & 0 & 0 \\
1 & - & 1 & 1 & 1 & 1 \\
0 & 0 & - & 0 & 0 & 0 \\
0 & 0 & 1 & - & 0 & 0 \\
0 & 0 & 0 & 0 & - & 0 \\
0 & 0 & 1 & 0 & 1 & -
\end{array}\right) .
$$

(9) Figure 2 shows the preference relations between the banks.

From Figure 2, it is clear that $B_{2}$ is the best option (Tables 6 and 7).

\section{Comparison Analysis and Discussion}

This section gives a comparison of the developed Dombi AOs with $m$ F Hamacher AOs [27] and an $m$ F-ELECTRE-I model [46] to show their feasibility and practicality.

(1) We compare the results of developed $m \mathrm{~F}$ Dombi AOs with $m \mathrm{~F}$ Hamacher AOs [27]. The results computed by applying both operators in first application are explained by Table 6 and Figure 3. In a similar way, the results computed using both operators in second application are explained by $\mathrm{Ta}$ ble 7 and Figure 4. Clearly, the results of $m \mathrm{~F}$ Hamacher weighted average ( $m$ FHWA) and $m \mathrm{~F}$ Hamacher weighted geometric ( $m$ FHWG) operators are different from our newly constructed $m$ FDWA and $m$ FDWG operators. The results of $m$ FHWA and $m$ FHWG operators are the same. Therefore, our developed AOs are more generalized and versatile than some existing models to handle $m \mathrm{~F}$ MCDM problems.

(2) From the second application, it can be observed that the final rankings by applying the $m$ FDWA and $m$ FDWG operators are $B_{2}>B_{6}>B_{4}>B_{1}>B_{5}>B_{3}$ and $B_{3}>B_{1}>B_{5}>B_{4}>B_{6}>B_{2}$, respectively. However, the final score values are not the same. When $m$ F-ELECTRE-I method is applied, the best option is $B_{2}$. Clearly, the optimal decision using $m$ F-ELECTRE-I method and $m$ FDWA operator is $B_{2}$.

(3) When a number of $m \mathrm{FNs}$ are aggregated with the help of $m$ FDWA and $m$ FDWG operators, different computations will increase rapidly. But our developed AOs can explain the assessed data more flexibly for decision making. The developed method ranks every objects in a given problem in comparison with $m$ F-ELECTRE-I approach [46].

6.1. Effectiveness Test. To examine the validity of the provided algorithm, we verify it with test criteria developed by Wang and Triantaphyllou [21] as follows (Tables 8 and 9):

(i) Test criterion I: if we change the membership grades of nonoptimal object with worse membership values

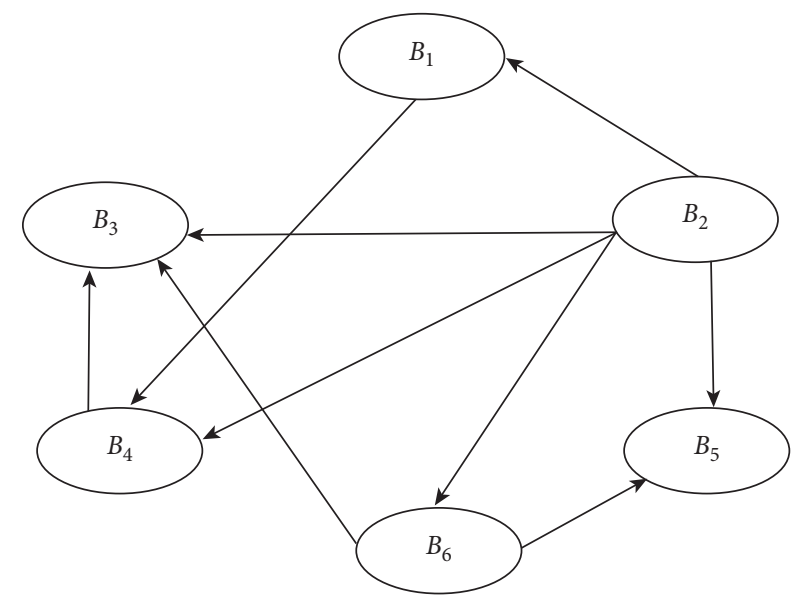

Figure 2: Outranking relation of banks.

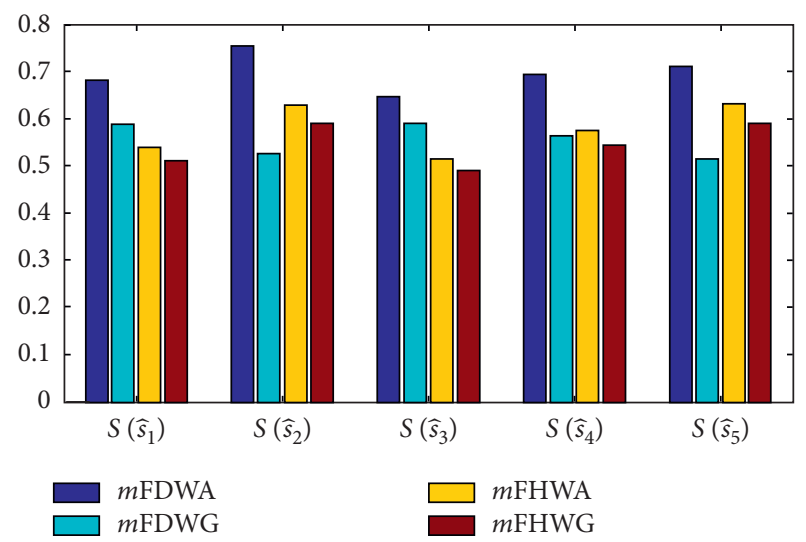

Figure 3: Comparison of first application in Section 5.1.

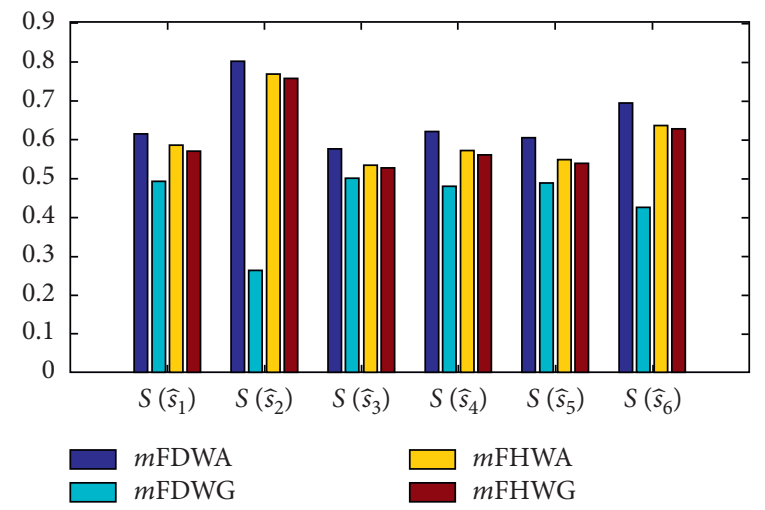

Figure 4: Comparison of second application in Section 5.2.

without effecting criteria, then the optimal object should not change.

(ii) Test criterion II: MCDM approach should satisfy transitive property.

(iii) Test criterion III: when a designated problem is resolved into different small issues and the similar 
TABle 6: Comparison of $m$ F Dombi AOs with $m$ F Hamacher AOs [27] in agriculture land selection.

\begin{tabular}{|c|c|c|c|c|c|c|}
\hline $\mathrm{AO}$ & $S\left(\widehat{s}_{1}\right)$ & $S\left(\widehat{s}_{2}\right)$ & $S\left(\widehat{s}_{3}\right)$ & $S\left(\widehat{s}_{4}\right)$ & $S\left(\widehat{s}_{5}\right)$ & Ranking order \\
\hline Proposed $m$ FDWA & 0.6814 & 0.7546 & 0.6459 & 0.6968 & 0.7108 & $Y_{2}>Y_{5}>Y_{4}>Y_{1}>Y_{3}$ \\
\hline Proposed $m$ FDWG & 0.5882 & 0.5266 & 0.5907 & 0.5643 & 0.5154 & $Y_{3}>Y_{1}>Y_{4}>Y_{2}>Y_{5}$ \\
\hline$m$ FHWA [27] & 0.5403 & 0.6287 & 0.5151 & 0.5725 & 0.6327 & $Y_{5}>Y_{2}>Y_{4}>Y_{1}>Y_{3}$ \\
\hline$m$ FHWG [27] & 0.5084 & 0.5881 & 0.4908 & 0.5445 & 0.5909 & $Y_{5}>Y_{2}>Y_{4}>Y_{1}>Y_{3}$ \\
\hline
\end{tabular}

TABle 7: Comparison of $m$ F Dombi AOs with $m$ F Hamacher AOs [27] in bank selection.

\begin{tabular}{|c|c|c|c|c|c|c|c|}
\hline $\mathrm{AO}$ & $S\left(\widehat{s}_{1}\right)$ & $S\left(\widehat{s}_{2}\right)$ & $S\left(\widehat{s}_{3}\right)$ & $S\left(\widehat{s}_{4}\right)$ & $S\left(\widehat{s}_{5}\right)$ & $S\left(\widehat{s}_{6}\right)$ & Ranking order \\
\hline Proposed $m$ FDWA & 0.6152 & 0.8007 & 0.5762 & 0.6191 & 0.6053 & 0.6945 & $B_{2}>B_{6}>B_{4}>B_{1}>B_{5}>B_{3}$ \\
\hline Proposed $m$ FDWG & 0.4929 & 0.2652 & 0.5008 & 0.4808 & 0.4868 & 0.4269 & $B_{3}>B_{1}>B_{5}>B_{4}>B_{6}>B_{2}$ \\
\hline$m$ FHWA [27] & 0.5851 & 0.7681 & 0.5342 & 0.5727 & 0.5490 & 0.6355 & $B_{2}>B_{6}>B_{1}>B_{4}>B_{5}>B_{3}$ \\
\hline$m$ FHWG [27] & 0.5711 & 0.7592 & 0.5267 & 0.5611 & 0.5381 & 0.6262 & $B_{2}>B_{6}>B_{1}>B_{4}>B_{5}>B_{3}$ \\
\hline
\end{tabular}

(1) Input:

$\widehat{S}$, an $m$ F decision matrix having $k$ objects and $n$ attributes.

$\Theta=\left(\Theta_{1}, \Theta_{2}, \ldots, \Theta_{n}\right)$, the vector having weights.

(2) Apply the $m$ FDWA operators to aggregate the data in $m$ F decision matrix $\widehat{S}$ and calculate the preference values $\widehat{s}_{i}$, where " $i$ " varies from 1 to $k$ for the $m \mathrm{FNs} \zeta_{i}$.

$\widehat{s}_{i}=\operatorname{mFDWA}_{\Theta}\left(\widehat{\zeta}_{i 1}, \widehat{\zeta}_{i 2}, \ldots, \widehat{\zeta}_{i n}\right)=\oplus_{j=1}^{n}\left(\Theta_{j} \widehat{\zeta}_{i j}\right)$

$=\left(1-\left(1 / 1+\left\{\sum_{j=1}^{n} \Theta_{j}\left(p_{1} \circ \zeta_{i j} / 1-p_{1} \circ \zeta_{i j}\right)^{k}\right\}^{1 / k}\right), \ldots, 1-\left(1 / 1+\left\{\sum_{j=1}^{n} \Theta_{j}\left(p_{m} \circ \zeta_{i j} / 1-p_{m} \circ \zeta_{i j}\right)^{k}\right\}^{1 / k}\right)\right)$.

When we use $m$ FDWG operators,

$\widehat{s}_{i}=m \operatorname{FDWG}_{\Theta}\left(\widehat{\zeta}_{i 1}, \widehat{\zeta}_{i 2}, \ldots, \widehat{\zeta}_{i n}\right)=\otimes_{j=1}^{n}\left(\widehat{\zeta}_{i j}\right)^{\Theta}$,

$=\left(1-\left(1 / 1+\left\{\sum_{j=1}^{n} \Theta_{j}\left(1-p_{1} \circ \zeta_{i j} / p_{1} \circ \zeta_{i j}\right)^{k}\right\}^{1 / k}\right), \ldots, 1-\left(1 /\left\{\sum_{j=1}^{n} \Theta_{j}\left(1-p_{m} \circ \zeta_{i j} / p_{m} \circ \zeta_{i j}\right)^{k}\right\}\right)\right)$.

(3) Compute the score values $S\left(\widehat{s}_{i}\right), i=1,2, \ldots, k$.

(4) Rank the objects $u_{i}, i=1,2, \ldots, k$ with respect to their scores $S\left(\widehat{s}_{i}\right)$. When the scores of two objects are equal, we apply the accuracy function to find the order of alternatives.

Output: the object containing maximum score value in last step will be the decision.

Algorithm 1: Computing maximum score value.

MCDM technique has been utilized, then the rank order of the objects should be similar to the original ranking order. We have checked these test criteria on developed MCDM approach under $m \mathrm{~F}$ Dombi AOs as below.

(1) Effectiveness test by criterion I: using this test, if we change only the membership grades of alternative $Y_{3} \quad$ with $\quad Y_{3}^{\prime}=(0.3,0.6,0.2),(0.4,0.3,0.2)$, $(0.5,0.7,0.3),(0.5,0.1,0.6)$ in Table 1 (that is, $3 \mathrm{~F}$ decision matrix), then the new $3 \mathrm{~F}$ decision matrix is shown in Table 8 . By using $m$ FDWA operator, the score values of the alternative are $S\left(\widehat{s}_{1}\right)=$ $0.6814, S\left(\widehat{s}_{2}\right)=0.7546, S\left(\widehat{s}_{3}^{\prime}\right)=0.6459, \quad S\left(\widehat{s}_{4}\right)=$ 0.6968, $S\left(\widehat{s}_{5}\right)=0.7108$. Clearly, $S\left(\widehat{s}_{2}\right)>S\left(\widehat{s}_{5}\right)>$ $S\left(\widehat{s}_{4}\right)>S\left(\widehat{s}_{1}\right)>S\left(\widehat{s}_{3}^{\prime}\right)$; consequently, the ranking of the objects is $Y_{2}>Y_{5}>Y_{4}>Y_{1}>Y_{3}^{\prime}$. Thus, $Y_{2}$ is the best alternative. According to above information, the presented AOs have been employed, and the decision alternative is $Y_{2}$ which is similar to the original optimal object. Similarly, if we change the membership grades of alternative $Y_{4}$ with $Y_{4}^{\prime}=(0.4,0.5,0.2),(0.7,0.5,0.5),(0.8,0.7$,
$0.1),(0.8,0.2,0.5)$ in Table 1 (that is, $3 \mathrm{~F}$ decision matrix), then the new $3 \mathrm{~F}$ decision matrix is shown in Table 9. By applying the $m$ FDWA operator, the ranking order of the alternatives is $Y_{2}>Y_{5}>Y_{1}>Y_{3}>Y_{4}^{\prime}$. Thus, the optimal alternative is $Y_{2}$ which is same as that of the original ranking. Therefore, the proposed algorithm is feasible under test criterion I.

(2) Effectiveness test by criteria II and III: based upon these test criteria, if we dissolved the designated problem (Application 1) into the subissues $\left\{Y_{1}, Y_{2}, Y_{3}\right\},\left\{Y_{2}, Y_{3}, Y_{4}\right\},\left\{Y_{3}, Y_{4}, Y_{5}\right\}$, and $\left\{Y_{4}, Y_{5}, Y_{1}\right\}$ and employed the procedure steps of Algorithm 1, then we obtain the ranking of these smaller issues as $Y_{2}>Y_{1}>Y_{3}, \quad Y_{2}>Y_{4}>Y_{3}$, $Y_{5}>Y_{4}>Y_{3}$, and $Y_{5}>Y_{4}>Y_{1}$, respectively. Hence, by uniting above criteria II and III, we obtain the overall ranking order of the alternatives as $Y_{2}>Y_{5}>Y_{4}>Y_{1}>Y_{3}$, which is exactly same as the original ranking order. Therefore, the developed algorithm is feasible under test criteria II and III. 
TABLE 8: 3F decision matrix.

\begin{tabular}{ccccc}
\hline & $S_{1}$ & $S_{2}$ & $S_{3}$ & $S_{4}$ \\
\hline$Y_{1}$ & $(0.7,0.6,0.3)$ & $(0.5,0.7,0.2)$ & $(0.6,0.3,0.7)$ & $(0.9,0.5,0.4)$ \\
$Y_{2}$ & $(0.9,0.6,0.5)$ & $(0.8,0.5,0.3)$ & $(0.4,0.5,0.8)$ & $(0.5,0.8,0.5)$ \\
$Y_{3}^{\prime}$ & $(0.3,0.6,0.2)$ & $(0.4,0.3,0.2)$ & $(0.5,0.7,0.3)$ & $(0.5,0.1,0.6)$ \\
$Y_{4}$ & $(0.5,0.6,0.3)$ & $(0.8,0.6,0.5)$ & $(0.7,0.8,0.2)$ & $(0.9,0.3,0.7)$ \\
$Y_{5}$ & $(0.9,0.7,0.6)$ & $(0.8,0.4,0.3)$ & $(0.6,0.5,0.7)$ & $(0.4,0.6,0.5)$ \\
\hline
\end{tabular}

TABLE 9: 3F decision matrix.

\begin{tabular}{ccccc}
\hline & $S_{1}$ & $S_{2}$ & $S_{3}$ & $S_{4}$ \\
\hline$Y_{1}$ & $(0.7,0.6,0.3)$ & $(0.5,0.7,0.2)$ & $(0.6,0.3,0.7)$ & $(0.9,0.5,0.4)$ \\
$Y_{2}$ & $(0.9,0.6,0.5)$ & $(0.8,0.5,0.3)$ & $(0.4,0.5,0.8)$ & $(0.5,0.8,0.5)$ \\
$Y_{3}$ & $(0.3,0.6,0.2)$ & $(0.4,0.3,0.2)$ & $(0.5,0.7,0.3)$ & $(0.5,0.1,0.6)$ \\
$Y_{4}^{\prime}$ & $(0.4,0.5,0.2)$ & $(0.7,0.5,0.5)$ & $(0.8,0.7,0.1)$ & $(0.8,0.2,0.5)$ \\
$Y_{5}$ & $(0.9,0.7,0.6)$ & $(0.8,0.4,0.3)$ & $(0.6,0.5,0.7)$ & $(0.4,0.6,0.5)$ \\
\hline
\end{tabular}

\section{Conclusions and Future Directions}

Aggregation operators are mathematical functions and essential tools of unifying several inputs into single valuable output. Due to the existence of multipolar data and multiple attributes in many real-world problems, classical MCDM methods are not useful to tackle complicated decision-making situations. To overcome the difficulties of existing models, we have combined $m$ FNs with Dombi AOs.

In this article, we have discussed MCDM issues based on $m \mathrm{~F}$ information. Motivated by Dombi operations, we have proposed certain $m \mathrm{~F}$ Dombi AOs, namely, $m$ FDWA, $m$ FDOWA, $m$ FDHA, $m$ FDWG, $m$ FDOWG, and $m$ FDHG operators. We have investigated different features of these operators. We have employed these AOs to enlarge the applicability scope of MCDM. We have given real-life applications for the selection of best agricultural land and for the selection of best bank regarding performance. At the end, we have provided a comparison of developed AOs with $m$ F-ELECTRE-I method [46] and $m$ F Hamacher AOs [27] and have authenticated the proposed strategy by effectiveness tests to check its validity. In the comparison, we have seen that the optimal alternative is the same by applying $m$ F-ELECTRE-I method [46], $m$ F Hamacher AOs [27], and proposed $m$ FDWA operator. However, it is different in case of $m$ FDWG operator. In the future, we plan to extend our work to (i) $m \mathrm{~F}$ Dombi prioritized AOs, (ii) $m \mathrm{~F}$ soft Dombi AOs, (iii) q-rung orthopair fuzzy Dombi AOs, and (iv) $q$-rung orthopair fuzzy soft Dombi AOs.

\section{Data Availability}

No data were used to support this study.

\section{Conflicts of Interest}

The authors declare that they have no conflicts of interest regarding the publication of the research article.

\section{Acknowledgments}

The authors extend their appreciation to the Deanship of Scientific Research at Majmaah University for funding this work under Project Number No. (RGP-2019- 5).

\section{References}

[1] K. T. Atanassov, "Intuitionistic fuzzy sets," Fuzzy Sets and Systems, vol. 20, no. 1, pp. 87-96, 1986.

[2] L. A. Zadeh, "Fuzzy sets," Information and Control, vol. 8, no. 3, pp. 338-353, 1965.

[3] R. R. Yager, "On ordered weighted averaging aggregation operators in multicriteria decisionmaking," IEEE Transactions on Systems, Man, and Cybernetics, vol. 18, no. 1, pp. 183-190, 1988.

[4] Z. Xu, "Intuitionistic fuzzy Aggregation operators," IEEE Transactions on Fuzzy Systems, vol. 15, no. 6, pp. 1179-1187, 2007.

[5] Z. Xu and R. R. Yager, "Some geometric Aggregation operators based on intuitionistic fuzzy sets," International Journal of General Systems, vol. 35, no. 4, pp. 417-433, 2006.

[6] W. R. Zhang, "Bipolar fuzzy sets and relations. A computational framework for cognitive modeling and multiagent decision analysis," in Proceedings of the First International Joint Conference of the North American Fuzzy Information Processing Society Biannual Conference. The Industrial Fuzzy Control and Intellige, IEEE, pp. 305-309, Vancouver, British Columbia, Canada, July 1994.

[7] G. Wei, F. E. Alsaadi, T. Hayat, and A. Alsaedi, "Bipolar fuzzy Hamacher Aggregation operators in multiple attribute decision making," International Journal of Fuzzy Systems, vol. 20, no. 1, pp. 1-12, 2018.

[8] H. Gao, G. Wei, and Y. Huang, "Dual hesitant bipolar fuzzy Hamacher prioritized Aggregation operators in multiple attribute decision making," IEEE Access, vol. 6, pp. 1150811522, 2018.

[9] P. Liu, "Some Hamacher Aggregation operators based on the interval-valued intuitionistic fuzzy numbers and their application to group decision making," IEEE Transactions on Fuzzy Systems, vol. 22, no. 1, pp. 83-97, 2013.

[10] C. Jana, M. Pal, and J.-q. Wang, "Bipolar fuzzy Dombi aggregation operators and its application in multiple-attribute decision-making process," Journal of Ambient Intelligence and Humanized Computing, vol. 10, no. 9, pp. 3533-3549, 2019.

[11] C. Jana, M. Pal, and J.-q. Wang, "Bipolar fuzzy Dombi prioritized Aggregation operators in multiple attribute decision making," Soft Computing, vol. 24, no. 5, pp. 3631-3646, 2020.

[12] X. He, "Typhoon disaster assessment based on Dombi hesitant fuzzy information aggregation operators," Natural Hazards, vol. 90, no. 3, pp. 1153-1175, 2018.

[13] X.-R. Xu and G.-W. Wei, "Dual hesitant bipolar fuzzy Aggregation operators in multiple attribute decision making," International Journal of Knowledge-Based and Intelligent Engineering Systems, vol. 21, no. 3, pp. 155-164, 2017.

[14] Z. Xu, "Approaches to multiple attribute group decision making based on intuitionistic fuzzy power Aggregation operators," Knowledge-Based Systems, vol. 24, no. 6, pp. 749-760, 2011.

[15] S. Xiao, "Induced interval-valued intuitionistic fuzzy Hamacher ordered weighted geometric operator and their application to multiple attribute decision making," Journal of Intelligent \& Fuzzy Systems, vol. 27, no. 1, pp. 527-534, 2014. 
[16] J. Chen and J. Ye, "Some single-valued neutrosophic Dombi weighted aggregation operators for multiple attribute decision-making," Symmetry, vol. 9, no. 82, pp. 1-11, 2017.

[17] H. Garg, "Generalised Pythagorean fuzzy geometric interactive Aggregation operators using Einstein operations and their application to decision making," Journal of Experimental \& Theoretical Artificial Intelligence, vol. 30, no. 6, pp. 763-794, 2018.

[18] M. Akram, W. A. Dudek, and J. M. Dar, "Pythagorean Dombi fuzzy aggregation operators with application in multicriteria decision-making," International Journal of Intelligent Systems, vol. 34, no. 11, pp. 3000-3019, 2019.

[19] G. Shahzadi, M. Akram, and A. N. Al-Kenani, "Decisionmaking approach under Pythagorean fuzzy Yager weighted operators," Mathematics, vol. 8, no. 1, p. 70, 2020.

[20] X. Peng and Y. Yang, "Fundamental properties of intervalvalued Pythagorean fuzzy aggregation operators," International Journal of Intelligent Systems, vol. 31, no. 5, pp. 444-487, 2016.

[21] J. Wang, G. Wei, J. Lu et al., "Some q-rung orthopair fuzzy Hamy mean operators in multiple attribute decision-making and their application to enterprise resource planning systems selection," International Journal of Intelligent Systems, vol. 34, no. 10, pp. 2429-2458, 2019.

[22] R. Arora and H. Garg, "Robust Aggregation operators for multi-criteria decision-making with intuitionistic fuzzy soft set environment," Scientia Iranica, vol. 25, no. 2, pp. 931-942, 2018.

[23] L. Wang and N. Li, "Pythagorean fuzzy interaction power Bonferroni mean aggregation operators in multiple attribute decision making," International Journal of Intelligent Systems, vol. 35, no. 1, pp. 150-183, 2020.

[24] F. Chiclana, F. Herrera, and E. Herrera-Viedma, "The ordered weighted geometric operator: properties and application in MCDM problems," in Technologies for Constructing Intelligent Systems, vol. 2, pp. 173-183, Physica, Heidelberg, Germany, 2002.

[25] D. Liang, Y. Zhang, Z. Xu, and A. P. Darko, "Pythagorean fuzzy Bonferroni mean aggregation operator and its accelerative calculating algorithm with the multithreading," International Journal of Intelligent Systems, vol. 33, no. 3, pp. 615-633, 2018.

[26] J. Chen, S. Li, S. Ma, and X. Wang, " $m$-polar fuzzy sets: an extension of bipolar fuzzy sets," The Scientific World Journal, vol. 2014, Article ID 416530, 8 pages, 2014.

[27] N. Waseem, M. Akram, and J. C. R. Alcantud, "Multi-attribute decision-making based on m-polar fuzzy Hamacher aggregation operators," Symmetry, vol. 11, no. 12, p. 1498, 2019.

[28] A. Khameneh and A. Kiliçman, "m-Polar fuzzy soft weighted aggregation operators and their applications in group decision-making," Symmetry, vol. 10, no. 11, p. 636, 2018.

[29] M. Akram, m-polar Fuzzy Graphs, Studies in Fuzziness and Soft Computing, p. 371, Springer, Berlin, Germany, 2019.

[30] M. Akram, A. Adeel, and J. C. R. Alcantud, "Multi-criteria group decision-making using an m-polar hesitant fuzzy TOPSIS approach,” Symmetry, vol. 11, no. 6, p. 795, 2019.

[31] A. Adeel, M. Akram, and A. N. A. Koam, "Group decisionmaking based on m-polar fuzzy Linguistic TOPSIS method," Symmetry, vol. 11, no. 6, p. 735, 2019.

[32] M. Akram, Shumaiza, and M. Arshad, "Bipolar fuzzy TOPSIS and bipolar fuzzy ELECTRE-I methods to diagnosis," Computational and Applied Mathematics, vol. 39, pp. 1-21, 2020.
[33] M. Akram, F. Ilyas, and H. Garg, "Multi-criteria group decision making based on ELECTRE I method in Pythagorean fuzzy information," Soft Computing, vol. 24, no. 5, pp. 3425-3453, 2020.

[34] G. Beliakov, A. Pradera, and T. Calvo, Aggregation Functions: A Guide for Practitioners, p. 221, Springer, Berlin, Germany, 2007.

[35] H. Garg and Nancy, "Linguistic single-valued neutrosophic prioritized Aggregation operators and their applications to multiple-attribute group decision-making," Journal of Ambient Intelligence and Humanized Computing, vol. 9, no. 6, pp. 1975-1997, 2018.

[36] H. Hamachar, "Uber logische verknunpfungenn unssharfer Aussagen und deren Zugenhorige Bewertungsfunktione Trappl," in Progress in Cybernatics and Systems Research, R. Klir, Ed., vol. 3pp. 276-288, 1978.

[37] P. P. Li, "Global implications of the indigenous epistemological system from the east," Cross Cultural \& Strategic Management, vol. 23, no. 1, pp. 42-77, 2016.

[38] W. Li, "Approaches to decision making with interval-valued intuitionistic fuzzy information and their application to enterprise financial performance assessment," Journal of Intelligent \& Fuzzy Systems, vol. 27, no. 1, pp. 1-8, 2014.

[39] X. Peng and Y. Yang, "Some results for Pythagorean fuzzy sets," International Journal of Intelligent Systems, vol. 30, no. 11, pp. 1133-1160, 2015.

[40] X. Peng and G. Selvachandran, "Pythagorean fuzzy set: state of the art and future directions," Artificial Intelligence Review, vol. 52, no. 3, pp. 1873-1927, 2019.

[41] X. Peng and J. Dai, "A bibliometric analysis of neutrosophic set: two decades review from 1998 to 2017," Artificial Intelligence Review, vol. 53, no. 2020, pp. 199-255.

[42] X. Wang and E. Triantaphyllou, "Ranking irregularities when evaluating alternatives by using some ELECTRE methods," Omega, vol. 36, no. 1, pp. 45-63, 2008.

[43] Z. S. Xu and Q. L. Da, "An overview of operators for aggregating information," International Journal of Intelligent Systems, vol. 18, no. 9, pp. 953-969, 2003.

[44] S. Zeng and W. Su, "Intuitionistic fuzzy ordered weighted distance operator," Knowledge-Based Systems, vol. 24, no. 8, pp. 1224-1232, 2011.

[45] L. Zhou, X. Zhao, and G. Wei, "Hesitant fuzzy Hamacher Aggregation operators and their application to multiple attribute decision making," Journal of Intelligent \& Fuzzy Systems, vol. 26, no. 6, pp. 2689-2699, 2014.

[46] M. Akram, N. Waseem, and P. Liu, "Novel approach in decision making with m-polar fuzzy ELECTRE-I," International Journal of Fuzzy Systems, vol. 21, no. 4, pp. 1117-1129, 2019.

[47] J. Dombi, "A general class of fuzzy operators, the DeMorgan class of fuzzy operators and fuzziness measures induced by fuzzy operators," Fuzzy Sets and Systems, vol. 8, no. 2, pp. 149-163, 1982. 